\title{
Exploring non-holomorphic soft terms in the framework of gauge mediated supersymmetry breaking
}

\author{
Utpal Chattopadhyay, ${ }^{a}$ Debottam Das ${ }^{b, c}$ and Samadrita Mukherjee ${ }^{a}$ \\ ${ }^{a}$ Department of Theoretical Physics, Indian Association for the Cultivation of Science, \\ $2 A \&$ B Raja S.C. Mullick Road, Jadavpur, Kolkata 700 032, India \\ ${ }^{b}$ Institute of Physics, \\ Bhubaneswar 751005, India \\ ${ }^{c}$ Homi Bhabha National Institute, Training School Complex, \\ Anushakti Nagar, Mumbai 400085, India \\ E-mail: tpuc@iacs.res.in, debottam@iopb.res.in, tpsm9@iacs.res.in
}

ABSTRACT: It is known that in the absence of a gauge singlet field, a specific class of supersymmetry (SUSY) breaking non-holomorphic (NH) terms can be soft breaking in nature so that they may be considered along with the Minimal Supersymmetric Standard Model (MSSM) and beyond. There have been studies related to these terms in minimal supergravity based models. Consideration of an F-type SUSY breaking scenario in the hidden sector with two chiral superfields however showed Planck scale suppression of such terms. In an unbiased point of view for the sources of SUSY breaking, the NH terms in a phenomenological MSSM (pMSSM) type of analysis showed a possibility of a large SUSY contribution to muon $g-2$, a reasonable amount of corrections to the Higgs boson mass and a drastic reduction of the electroweak fine-tuning for a higgsino dominated $\widetilde{\chi}_{1}^{0}$ in some regions of parameter space. We first investigate here the effects of the NH terms in a low scale SUSY breaking scenario. In our analysis with minimal gauge mediated supersymmetry breaking (mGMSB) we probe how far the results can be compared with the previous pMSSM plus NH terms based study. We particularly analyze the Higgs, stop and the electroweakino sectors focusing on a higgsino dominated $\widetilde{\chi}_{1}^{0}$ and $\widetilde{\chi}_{1}^{ \pm}$, a feature typically different from what appears in mGMSB. The effect of a limited degree of RG evolutions and vanishing of the trilinear coupling terms at the messenger scale can be overcome by choosing a non-minimal GMSB scenario, such as one with a matter-messenger interaction.

KeYwords: Supersymmetry Phenomenology

ARXIV EPrint: 1710.10120 


\section{Contents}

1 Introduction 1

2 Gauge mediated SUSY breaking and non-holomorphic soft terms $\quad 3$

$\begin{array}{llr}3 & \text { Results } & \mathbf{7}\end{array}$

3.1 Effects on top-squarks and Higgs masses 8

$\begin{array}{ll}3.2 & \text { Phenomenological implications through the electroweakino sector } \\ & 12\end{array}$

$\begin{array}{lll}3.3 & \text { Higgsino like NLSP decays } & 14\end{array}$

$\begin{array}{lll}4 & \text { Conclusion } & 17\end{array}$

\section{Introduction}

The discovery of Higgs boson with a mass of $125 \mathrm{GeV}$ [1-3] along with null search results for supersymmetry (SUSY) at the Large Hadron Collider (LHC) $[4,5]$ has in general enhanced the masses of the superpartners of the low energy supersymmetry models. This is even more prominent in models like minimal supergravity (mSUGRA) [6-11] or Constrained Minimal Supersymmetric Standard Model (CMSSM) where correlations among the masses via renormalization group evolution (RGE) effects make the spectra further heavy. In the Minimal Supersymmetric Standard Model (MSSM) [12-19], unless the third generation scalars are themselves too massive, the Higgs mass data translates into the requirement of a large top-squark left-right (L-R) mixing. This is required for obtaining the desired amount of radiative corrections for the mass of the CP-even lighter Higgs boson $(h)$ [20]. This, on the other hand, increases the electroweak fine-tuning [21-35].

On the dark matter (DM) front, MSSM may provide with a suitable candidate like the lightest neutralino, the lightest supersymmetric particle (LSP) [36, 37]. The LSP in its bino dominated state needs very light sleptons to satisfy the DM relic density limits from PLANCK experiment [38]. Light sleptons is a rather disfavored scenario for most of the SUSY models in the post-Higgs discovery era. A bino can, however, be a favorable DM candidate, via its coannihilations with stau $\left(\tilde{\tau}_{1}\right)$ or via its s-channel higgs exchange self-annihilation mechanism [39-42]. In spite of the above, even with the possibility of having a reasonably light LSP, the above coannihilation or resonance annihilations of binos require stringent correlations among unrelated SUSY parameters. A mixed bino-higgsino scenario like the Hyperbolic Branch [35, 43, 44]/Focus Point [45-51] zones can still have a reasonably light LSP. However, it is not so favorable via the spin-independent (SI) direct detection cross-section limits [52]. The reason lies in the larger values of Higgs- $\widetilde{\chi}_{1}^{0}-\widetilde{\chi}_{1}^{0}$ couplings. On the other hand, in MSSM one can have a higgsino like LSP as a suitable dark matter candidate that can pair-annihilate efficiently via their couplings to the $\mathrm{W} / \mathrm{Z}$ 
gauge bosons [53-55]. Unlike a bino dominated LSP, a higgsino type of DM would not require any fine-adjustment of uncorrelated SUSY parameters. But a higgsino satisfying the observational relic density limits is typically quite heavy [53-55] $(\sim 1 \mathrm{TeV})^{1}$ giving rise to a large electroweak fine-tuning in MSSM. It is thus desirable to have a model that has a higgsino like LSP but is able to guard fine-tuning to become large. We further note that a higgsino like LSP scenario of phenomenological MSSM (pMSSM) [57] is significantly free from the LHC bounds $[58,59]$.

The other issue at hand in MSSM is the stringent muon $g-2$ constraint [60-62]. Satisfying the muon $g-2$ constraint [63] in the MSSM is associated with a significant degree of reduction of parameter space. As we know, SUSY contributions to muon $g-2$ are enhanced when the lighter electroweakinos $\left(\widetilde{\chi}_{1}^{ \pm}\right.$and $\left.\widetilde{\chi}_{1}^{0}\right)$ or the lighter smuon mass $\tilde{\mu}_{1}$ become small. Concerning $\operatorname{Br}\left(B \rightarrow X_{s}+\gamma\right)$ [64], the constraint can be effective in MSSM for a high $\tan \beta$ [65-68], the ratio of Higgs vacuum expectation values and non-decoupled zone of squark masses.

All the above that restrict the MSSM parameter space may be addressed reasonably well when one includes non-holomorphic $(\mathrm{NH})$ soft terms on the top of the usual holomorphic soft terms of MSSM [69, 70]. Staying within an MSSM setup terms like $\phi^{2} \phi^{*}$ or a higgsino mass term like $\psi \psi$ can be soft SUSY breaking in nature if there is no presence of a gauge singlet field [71]. There have been several works over the past two decades [6982] that analyzed the effects of introducing $\mathrm{NH}$ soft terms. ${ }^{2}$ There are three distinct signatures i) the trilinear $\mathrm{NH}$ terms may enhance L-R mixing thus influencing various phenomenologies, ii) higgsinos get an additional soft-term source, iii) the Higgs potential remains unaffected at the tree level thus electroweak fine-tuning may hardly change. The latter effect can give rise to a valid higgsino dark matter with low fine-tuning, whereas the L-R mixing effect may contribute to $(g-2)_{\mu}, \operatorname{Br}\left(B \rightarrow X_{s}+\gamma\right)$, or the higgs mass radiative corrections $[69,70,78-81]$. The $\mathrm{NH}$ soft terms can also influence on the scalar potential terms involving colored/charged scalars [82]. Thus, there are associated charge and color breaking (CCB) minima constraints that one must take care of in the limiting cases of large trilinear $\mathrm{NH}$ couplings.

All the above effects in combination may potentially cause very distinct outcomes in global analyses of low energy SUSY models [84-87]. In a model-dependent standpoint, one finds that such NH soft terms may arise out of interactions like $\frac{1}{M^{3}}\left[X X^{*} \Phi^{2} \Phi^{*}\right]_{D}$ and $\frac{1}{M^{3}}\left[X X^{*} D^{\alpha} \Phi D_{\alpha} \Phi\right]_{D}[71]$. In the above one considers a scenario where supersymmetry is broken in the hidden sector via an auxiliary field $F$ of the chiral superfield $X$. $\Phi$ is another chiral superfield and $M$ refers to the mediation scale. Both these terms have a strength $\frac{|F|^{2}}{M^{3}} \sim \frac{M_{W}^{2}}{M}[70]$. Clearly, there are large suppressions $(\sim 1 / M)$ for the NH soft terms in supergravity scenarios where $M$ can be very large nearing the Planck scale. However, the suppression effects may be small if one considers a SUSY breaking mechanism that would allow a low mediation scale.

With the above motivation, we like to explore the effects of having NH soft terms in gauge mediated supersymmetry breaking (GMSB) where SUSY breaking may occur at a

\footnotetext{
${ }^{1}$ The limit goes below $600 \mathrm{GeV}$ for higgsinos undergoing sfermion coannihilations [56].

${ }^{2}$ See ref. [83] for an analysis with hard SUSY breaking terms.
} 
smaller scale and the scale of mediation, the messenger scale is also low. We will try to gauge, how far the electroweak scale analysis with $\mathrm{NH}$ soft terms of ref. [70] referred as Non-Holomorphic Supersymmetric Standard Model (NHSSM) preserves its conclusion in a minimal GMSB (mGMSB) setup [88-95]. We remind that the mGMSB has gravitino as its dark matter candidate with its mass ranging from a few eV up to $\mathcal{O}(1) \mathrm{GeV}$ [94, 96-101] whereas the Next-to-lightest-SUSY Particle (NLSP) is largely a bino dominated neutralino. We will focus here on realizing an mGMSB setup with higgsino as the NLSP. This is unlike the typical case of a bino like NLSP that is characteristic of GMSB types of analyses [8894]. ${ }^{3}$ We will investigate the effects of NH soft terms on NLSP decaying to a gravitino and a Z-boson $\widetilde{\chi}_{1}^{0} \rightarrow \widetilde{G}+Z$ or $\widetilde{\chi}_{1}^{0} \rightarrow \widetilde{G}+h[94,98]$ while assuming the other higgs bosons to be much heavier than the NLSP.

We will now briefly describe the plan of the work. In section 2 we will outline the gauge mediated breaking SUSY mechanism and introduce non-holomorphic soft terms within the above framework. We will discuss the effect of NH terms on Higgs and electroweakinos. In section 3 we will describe the results of the relevant parameter scanning on the top-squark and higgs boson masses and investigate the phenomenologies involving $\operatorname{Br}\left(B \rightarrow X_{s}+\gamma\right)$ and muon $g-2$. We will compare our results with the scenario of MSSM with NH terms where one gives all the input parameters at the electroweak scale. Furthermore, focusing on a parameter space where the NLSP is a higgsino dominated lightest neutralino, a scenario typically unavailable in mGMSB, we will estimate the relevant NLSP decay widths for a higgsino producing gravitino and other particles like $h$ and $Z$ bosons. Finally, we will conclude in section 4 .

\section{Gauge mediated SUSY breaking and non-holomorphic soft terms}

In the Gauge-Mediated-SUSY breaking (GMSB) Models [88-94] SUSY is broken in a hidden/secluded sector. In the absence of the knowledge of the hidden sector, one may consider a spurion $S$ which is a chiral superfield, singlet under the SM group. SUSY breaking in this sector is realized via $S$ acquiring a vacuum expectation value (vev) via its scalar and auxiliary components. Thus,

$$
\langle S\rangle=M+\theta \theta\langle F\rangle .
$$

The parameters $M$ and $\sqrt{\langle F\rangle} \equiv \sqrt{F}$ are the fundamental scales related to GMSB. Apart from the hidden sector and the observable sector where MSSM fields reside, there is a messenger sector that itself experiences the SUSY breaking and mediates the SUSY breaking it is affected with, to the observable sector. The messenger sector connects to the hidden sector via the singlet spurion field $S$ that goes into a superpotential containing superfields of the messenger and hidden sectors. This results into a SUSY breaking via the two vevs of the scalar and the auxiliary components of $S$ namely, $M$ and $\langle F\rangle$ causing a splitting of the masses of the messenger sector scalars $\left(m_{\phi, \tilde{\phi}}^{2}=M^{2} \pm\langle F\rangle\right)$ and fermions $\left(m_{\psi, \tilde{\psi}}=M\right)$. The fact that the scalars should not go tachyonic, so that the vacuum stability is unaffected

\footnotetext{
${ }^{3}$ Higgsino NSLP may be possible in an extended version of GMSB model that incorporates non-unified messenger sector and messenger-matter interactions [102].
} 
demands $\langle F\rangle<M^{2}{ }^{4}$ Considering a large $M$, we can integrate out the messenger scalars and fermions that are charged under the $\mathrm{SM}$ gauge group $\mathrm{SU}(3)_{C} \times \mathrm{SU}(2)_{L} \times \mathrm{U}(1)_{Y}$. The low energy effective theory below $M$ would then break supersymmetry in the observable sector. The observable sector soft terms like the masses and couplings are generated via messenger particles in loops. This ensures renormalizability, a positive feature of GMSB models. The MSSM soft terms thus obtained via gauge boson and gaugino interactions are also flavor blind. It is the messenger sector particles, assumed to be heavy for phenomenological reasons characterize the low energy phenomenology. The gaugino masses arise out of one-loop diagrams such as that involving a messenger scalar and a fermion. The scalar mass squares arise out of two loop diagrams that may include messenger scalars and fermions apart from gauge bosons and gauginos.

Thus, the gaugino masses $m_{\lambda}$ and scalar mass squares $m_{\tilde{f}}^{2}$ read,

$$
m_{\lambda} \sim \frac{g^{2}}{16 \pi^{2}} \Lambda\left(1+\mathcal{O}\left(\langle F\rangle^{2} / M^{4}\right)\right) ; \text { and, } \quad m_{\tilde{f}}^{2} \sim\left(\frac{g^{2}}{16 \pi^{2}}\right)^{2} \Lambda^{2}\left(1+\mathcal{O}\left(\langle F\rangle^{2} / M^{4}\right)\right)
$$

where $\Lambda=\frac{\langle F\rangle}{M}$, is the SUSY breaking scale in MSSM. The parameters $m_{\lambda}$ and $m_{\tilde{f}}$ come with similar values. The trilinear parameters are small and considered to be vanishing at the messenger scale, a result of the fact that the messengers can only interact with SM fields via gauge interactions. Vanishing of trilinear couplings at the messenger scale may also be seen in ref. [95] that used wavefunction renormalization method without a need of using Feynman diagrams in this regard. The trilinear couplings associated with the third generation of scalars are however non-vanishing at the electroweak scale via renormalization group evolutions. A soft mass value of $m_{\tilde{f}} \sim 1 \mathrm{TeV}$ would set $\frac{\langle F\rangle}{M} \sim 10^{5} \mathrm{GeV}$ (eq. (2.2)), though this neither specifies $\langle F\rangle$ nor $M$. We note that the scalars should not break vacuum stability, as mentioned before, demands $\langle F\rangle\left\langle M^{2}\right.$. In its limiting case of an equality, one has $\frac{\langle F\rangle}{M}=M=10^{5} \mathrm{GeV}$. This results into $M=10^{5} \mathrm{GeV}$ and $\sqrt{\langle F\rangle}=10^{5} \mathrm{GeV}$. Considering the inequality itself, one obtains the lower bounds $M>10^{5} \mathrm{GeV}$ and $\sqrt{\langle F\rangle}>$ $10^{5} \mathrm{GeV}$.

The upper limit of the messenger mass $M$ comes from the relative degree of strengths of the gauge and gravity mediations since the superfield $S$ may also cause gravity effects, though in much smaller strength, in addition to the SUSY breaking associated with a GMSB scenario. The scalar mass squares from the gravity and gauge mediated scenarios may now be compared. A representative factor of $1 / 1000$ for the ratio of the two soft mass squares would mean $\frac{g^{2}}{16 \pi^{2}} \frac{\langle F\rangle}{M}>10^{3 / 2} \frac{\langle F\rangle}{M_{P}}$, where $M_{P}$ is the Planck mass [94]. The above results into an approximate upper bound $M<10^{15} \mathrm{GeV} .{ }^{5}$ Correspondingly, for a $1 \mathrm{TeV}$ scalar mass, one finds $\sqrt{\langle F\rangle}<10^{10} \mathrm{GeV}$. Summarizing, one finds,

$$
10^{5}<\sqrt{\langle F\rangle}<10^{10} \mathrm{GeV} \text {, and, } 10^{5}<M<10^{15} \mathrm{GeV} .
$$

Considering the supergravity relation the gravitino mass is given by $m_{3 / 2}=\frac{F}{\sqrt{3} M_{P}}$. Depending on $\langle F\rangle$ (eq. (2.3)), the gravitino mass may range from $\sim 1 \mathrm{eV}$ to $\sim 1 \mathrm{GeV}$.

\footnotetext{
${ }^{4}$ Realistic scenarios rather satisfy $\langle F\rangle \ll M^{2}$.

${ }^{5}$ This is via considering a value of $g$ corresponding to the strong coupling at the electroweak scale.
} 
Now, within the MSSM framework the NH soft terms are given by [69, 70]: 6

$$
-\mathcal{L}_{\mathrm{soft}}^{\prime N H} \supset \tilde{Q} \cdot H_{d}^{c} A_{u}^{\prime} \tilde{U}+\tilde{Q} \cdot H_{u}^{c} A_{d}^{\prime} \tilde{D}+\tilde{L} \cdot H_{u}^{c} A_{e}^{\prime} \tilde{E}+\mu^{\prime} \tilde{H}_{u} \cdot \tilde{H}_{d}+\text { h.c. }
$$

Here, $A_{i}^{\prime}$ refers to three $3 \times 3$ matrices $\left(\left[A_{i}^{\prime}\right]\right)$ belonging to the family space of squarks/sleptons. We remind that the MSSM soft terms are generated in mGMSB via gauge and gaugino interactions that are flavor blind. The messenger sector particles occur only in loops. All the elements of $\left[A_{i}\right]$, the associated parameters for holomorphic trilinear interactions that arise at two-loop levels are considered to be zero at the messenger scale [17-19, 88-94]. We note that eq. (2.4) differs from the holomorphic trilinear soft SUSY breaking Lagrangian by a change in the Higgs fields. Thus, $H_{u, d}$ are replaced by their conjugates in eq. (2.4) with appropriate book-keeping for the associated scalar fields for hypercharge assignments. Hence, as with the elements of $\left[A_{i}\right]$, the same for $\left[A_{i}^{\prime}\right]$ should also vanish at the same scale. With a limited zone of RG running typical to a GMSB scenario both $\left[A_{i}\right]$ and $\left[A_{i}^{\prime}\right]$ terms are tiny at the electroweak scale for the first two generations. Thus $\left[A_{i}^{\prime}\right]$ terms do not cause any violation of flavor changing neutral current (FCNC) constraints that are typically stringent for observables related to the first two generations of fermions.

As mentioned in section 1, terms like $\phi^{2} \phi^{*}$ and a higgsino mass soft-term $\psi \psi$ (eq. (2.4)) may originate from D-term contributions like $\frac{1}{M^{3}}\left[X X^{*} \Phi^{2} \Phi^{*}\right]_{D}$ and $\frac{1}{M^{3}}\left[X X^{*} D^{\alpha} \Phi D_{\alpha} \Phi\right]_{D}[70,71]$. Since the terms are of strength $\frac{M_{W}^{2}}{M}$, a low mediation scale $M$ such as that appears in a GMSB scenario may be relevant for probing the phenomenological implications. The parameters for trilinear non-holomorphic soft terms are small, similar to the same of trilinear holomorphic terms at the mediation scale $M$. We will keep the other D-term soft breaking NH interaction namely the higgsino mass soft term characterized by $\mu^{\prime}$ (to be given at the scale $M$ ), to have an unknown SUSY breaking origin. ${ }^{7}$ This is considering the issue of an associated re-parametrization invariance of the higgsino mass soft term $[73,76,80,81]$. Reparametrization comes from unrelated quantities like $\mu$, the higgsino mixing superpotential parameter and the higgs scalar soft mass parameters $m_{H_{U}}^{2}$ and $m_{H_{D}}^{2}$. An assumption of an independent SUSY breaking mechanism to have a higgsino mass soft term essentially avoids such concerns (See discussions in [80, 81] along with the references therein).

Coming to the minimal GMSB, to preserve the gauge coupling unification one considers messengers to belong to a complete $\mathrm{SU}(5)$ representation or any other complete representation of a larger gauge group that includes $\mathrm{SU}(5)$ as a sub-group. With $S$ as the spurion field mentioned before one has the superpotential,

$$
W_{\text {mess }}=S \Phi \bar{\Phi}
$$

In the simplest case, there will be $N_{5}$ number of flavor of messenger copies $\Phi$ and $\bar{\Phi}$ transforming as 5 and $\overline{5}$ representations of SU(5). The soft SUSY breaking parameters like

\footnotetext{
${ }^{6}$ There can be an $\mathrm{NH}$ soft term like $\psi \lambda$ involving higgsinos and gauginos. This would however take us away from MSSM, hence ignored [71].

${ }^{7}$ Additionally, such a term cannot originate at a one-loop level in mGMSB, thus becoming suppressed.
} 
gaugino and scalar masses are given as follows.

$$
M_{\alpha}=\frac{g_{\alpha}^{2}}{16 \pi^{2}} N_{5} \Lambda g(x), \quad(\alpha=1,2,3) .
$$

Here $\alpha$ refers to $\mathrm{SM}$ gauge group $\left(\mathrm{U}(1)_{Y}, \mathrm{SU}(2)_{L}, \& \mathrm{SU}(3)_{c}\right)$. $x=\frac{\langle F\rangle}{M^{2}}, \Lambda=\frac{\langle F\rangle}{M}$ and $g(x)$ is given as,

$$
g(x)=\frac{1}{x^{2}}((1+x) \log (1+x)+(1-x) \log (1-x)) .
$$

The scalar masses are given by,

$$
m_{\tilde{f}}^{2}=2 \Lambda^{2} N_{5} \sum_{\alpha}\left(\frac{g_{\alpha}^{2}}{16 \pi^{2}}\right)^{2} C_{\alpha} f(x) .
$$

Here, $f(x)$ is given by,

$$
f(x)=\frac{1+x}{x^{2}}\left(\log (1+x)-2 \operatorname{Li}_{2}\left(\frac{x}{1+x}\right)+\frac{1}{2} \operatorname{Li}_{2}\left(\frac{2 x}{1+x}\right)\right)+(x \rightarrow-x) .
$$

$C_{\alpha}$ is the quadratic Casimir of the representation of the gauge group factor $G_{\alpha}$ under which the scalar field $\tilde{f}$ transforms. For the gauge groups involved, the Casimirs are: $C_{\mathrm{SU}(n)}=\frac{n^{2}-1}{2 n}$ and $C_{\mathrm{U}(1)}=(3 / 5) Y^{2}$. The Euler dilogarithm function $\operatorname{Li}_{2}(x)$ is given by $\operatorname{Li}_{2}(x)=-\int_{0}^{x} \frac{\log (1-t)}{t} d t$, for $0<x<1$. Under the assumption of $\langle F\rangle \ll M^{2}$ one has $g(x) \simeq 1$ and $f(x) \simeq 1$. This results into,

$$
M_{\alpha}=\frac{g_{\alpha}^{2}}{16 \pi^{2}} \Lambda N_{5}
$$

and,

$$
m_{\tilde{f}}^{2}=2 \Lambda^{2} N_{5} \sum_{\alpha}\left(\frac{g_{\alpha}^{2}}{16 \pi^{2}}\right)^{2} C_{\alpha} .
$$

The following are the model parameters where the NH higgsino soft parameter $\mu_{0}^{\prime}$ is the input value of $\mu^{\prime}$ at the messenger scale $M$ at which the common NH trilinear coupling parameter $A_{0}^{\prime}$ vanish.

$$
\Lambda, M, \tan \beta, N_{5}, \operatorname{sign}(\mu) \text {, and } \mu_{0}^{\prime} .
$$

The NH trilinear couplings of eq. (2.4) modify the off-diagonal elements of the scalar mass matrix as given below $[69,70]$.

$$
M_{\tilde{u}}^{2}=\left(\begin{array}{cc}
m_{\tilde{Q}}^{2}+\left(\frac{1}{2}-\frac{2}{3} \sin ^{2} \theta_{W}\right) M_{Z}^{2} \cos 2 \beta+m_{u}^{2} & -m_{u}\left(A_{u}-\left(\mu+A_{u}^{\prime}\right) \cot \beta\right) \\
-m_{u}\left(A_{u}-\left(\mu+A_{u}^{\prime}\right) \cot \beta\right) & m_{\tilde{u}}^{2}+\frac{2}{3} \sin ^{2} \theta_{W} M_{Z}^{2} \cos 2 \beta+m_{u}^{2}
\end{array}\right) .
$$

Similar mass matrices for sleptons (or a down-type of squarks) would have the off-diagonal term $-m_{e}\left(A_{e}-\left(\mu+A_{e}^{\prime}\right) \tan \beta\right)$. Clearly, the NH trilinear couplings contribute to the offdiagonal elements where $\mu$ is replaced by $\mu+A_{f}^{\prime}(f=u, d$, e etc.). The above indicates a more significant impact of L-R mixing for i) low values of $\tan \beta$ for the up type of squarks and ii) high values of $\tan \beta$ for down type of squarks or sleptons. 
Now, the discovery of the Higgs boson with mass of $125.09 \pm 0.24 \mathrm{GeV}[1-3]$ is translated into a large amount of radiative corrections to the mass of the lighter neutral CP-even Higgs boson $h$. The above requirement causes an increase in the masses of the top-squarks in MSSM and/or one needs an appropriately large value of $\left|A_{t}\right|$, so as to have a stronger $\tilde{t}_{L}-\tilde{t}_{R}$ mixing. Thus, the NH soft trilinear parameter $A_{t}^{\prime}$ that affects the L-R mixing has important contributions toward the above radiative corrections for small values of $\tan \beta$.

The lightest CP-even higgs boson mass up to one loop can be read as follows [20].

$$
m_{h, \text { top }}^{2}=m_{Z}^{2} \cos ^{2} 2 \beta+\frac{3 g_{2}^{2} \bar{m}_{t}^{4}}{8 \pi^{2} M_{W}^{2}}\left[\ln \left(\frac{m_{\tilde{t}_{1}} m_{\tilde{t}_{2}}}{\bar{m}_{t}^{2}}\right)+\frac{X_{t}^{\prime 2}}{m_{\tilde{t}_{1}} m_{\tilde{t}_{2}}}\left(1-\frac{X_{t}^{\prime 2}}{12 m_{\tilde{t}_{1}} m_{\tilde{t}_{2}}}\right)\right] .
$$

Here, $X_{t}^{\prime}=A_{t}-\left(\mu+A_{t}^{\prime}\right) \cot \beta$. Clearly, $A_{t}^{\prime}=0$ corresponds to the MSSM result. Here $\bar{m}_{t}$ refers to the running top-quark mass that includes corrections from the electroweak, quantum chromodynamics (QCD) and SUSY QCD related effects [103]. The maximal mixing scenario refers to $X_{t}^{\prime}=\sqrt{6} M_{S}$ where $M_{S}=\sqrt{m_{\tilde{t}_{1}} m_{\tilde{t}_{2}}}$. With a suitable $A_{t}^{\prime}$, it is possible to satisfy the Higgs mass constraint with a relatively smaller value of $\left|A_{t}\right|$ in NHSSM, compared to MSSM.

The changes in the neutralino and chargino mass matrices are as shown below. Essentially, $\mu$ is replaced by $\mu+\mu^{\prime}$ as in the tree level results.

$$
\begin{aligned}
M_{\widetilde{\chi^{0}}} & =\left(\begin{array}{cccc}
M_{1} & 0 & -M_{Z} \cos \beta \sin \theta_{W} & M_{Z} \sin \beta \sin \theta_{W} \\
0 & M_{2} & M_{Z} \cos \beta \cos \theta_{W} & -M_{Z} \sin \beta \cos \theta_{W} \\
-M_{Z} \cos \beta \sin \theta_{W} & M_{Z} \cos \beta \cos \theta_{W} & 0 & -\left(\mu+\mu^{\prime}\right) \\
M_{Z} \sin \beta \sin \theta_{W} & -M_{Z} \sin \beta \cos \theta_{W} & -\left(\mu+\mu^{\prime}\right) & 0
\end{array}\right) . \\
M_{\widetilde{\chi^{ \pm}}} & =\left(\begin{array}{cc}
M_{2} & \sqrt{2} M_{W} \sin \beta \\
\sqrt{2} M_{W} \cos \beta & \left(\mu+\mu^{\prime}\right)
\end{array}\right) .
\end{aligned}
$$

We note that the collider bound on the lighter chargino mass, except the issue of radiative corrections, will apply to essentially $\left|\mu+\mu^{\prime}\right|$ instead of $|\mu|$ in case $\widetilde{\chi}_{1}^{ \pm}$is higgsino dominated in nature. Since the Higgs potential is unaffected, the electroweak fine-tuning measure at tree level is still dependent on $\mu[70,80,81]$ rather than it has anything to do with $\mu^{\prime}$. A large higgsino mass with less electroweak fine-tuning becomes a possibility.

In contrast to the NHSSM that is based on electroweak scale inputs we should keep in mind that the dependence of relevant quantities especially $\mu$ on $\mu^{\prime}$ and $A_{t}^{\prime}$ can be significant in NHmGMSB due to RGE effects. Besides, it is important to mention that a fine-tuning measure analyzed in a scenario with $\mathrm{NH}$ terms in a predictive model that uses RGEs can be less independent with respect to the higgsino mass [81]. This is unlike what is seen in electroweak fine-tuning in NHSSM as mentioned above.

\section{Results}

We have realized the non-holomorphic MSSM on a GMSB setup, that is going to be referred as the NHmGMSB model, by using the codes SARAH-4.9.1 [104-107] and SPheno3.3.8 [108]. Two-loop RGEs of the MSSM soft parameters, plus the same at one-loop for 
the $\mathrm{NH}$ soft parameters are used to generate the sparticle mass spectra $[73,74,80,113]$. The codes use two-loop corrections for Higgs states $[114,115]$ and additionally compute all the relevant flavor observables [112]. As mentioned earlier, the free parameters that are to be scanned are

$$
\Lambda, M_{\text {mess }},{ }^{8} \tan \beta, N_{5}, \operatorname{sign}(\mu) \text { and } \mu_{0}^{\prime},
$$

where the number of the messenger copies is taken to be one $\left(N_{5}=1\right)$. At the messenger scale $M_{\text {mess }}$, the soft SUSY breaking mass parameters are given via eqs. (2.6), (2.8) and the values of (non) holomorphic trilinear coupling parameters $\left(A_{0}^{\prime}\right), A_{0}$ are taken to be zero. Choosing $\operatorname{sign}(\mu)=1$ we scan the following volume of the mGMSB parameters.

$$
\begin{aligned}
3.0 \times 10^{5} \mathrm{GeV} & \leqslant \Lambda \leqslant 1.0 \times 10^{6} \mathrm{GeV}, \\
2 \times 10^{6} \mathrm{GeV} & \leqslant M_{\text {mess }} \leqslant 10^{8} \mathrm{GeV}, \\
\tan \beta & =10 \text { and } 40 \\
-4000 \mathrm{GeV} & \leqslant \mu_{0}^{\prime} \leqslant 4000 \mathrm{GeV},
\end{aligned}
$$

with $m_{t}^{\text {pole }}=173.5 \mathrm{GeV}, m_{b}^{\overline{M S}}=4.18 \mathrm{GeV}$ and $m_{\tau}=1.77 \mathrm{GeV}[116]$ and $M_{\mathrm{SUSY}}=$ $\sqrt{m_{\tilde{t}_{1}} m_{\tilde{t}_{2}}}$. Additionally, we note that a higher value of $M_{\text {mess }}$ than what is given in eq. (3.2) would not be so consistent with our motivation of choosing a low scale SUSY breaking model like mGMSB. This is in keeping with limiting the mediation scale suppression of the NH soft terms as mentioned in section 1 . On the other hand, reducing $M_{\text {mess }}$ further than the lower limit of eq. (3.2) would hardly provide with a reasonable range of RG running of both $A_{t}$ and $A_{t}^{\prime}$ that is essential for satisfying the higgs mass data (without trying to compensate it via choosing a larger value of $\Lambda$ ).

The SUSY Higgs mass $\left(m_{h}\right)$ limits [116] and the constraints from B-physics namely $B \rightarrow X_{s}+\gamma, B_{s} \rightarrow \mu^{+} \mu^{-}$are mentioned as below [116].

$$
\begin{aligned}
122.1 \mathrm{GeV} & \leqslant m_{h} \leqslant 128.1 \mathrm{GeV}, \\
2.99 \times 10^{-4} & \leqslant B r\left(B \rightarrow X_{s}+\gamma\right) \leqslant 3.87 \times 10^{-4}(2 \sigma), \\
1.5 \times 10^{-9} & \leqslant B r\left(B_{s} \rightarrow \mu^{+} \mu^{-}\right) \leqslant 4.3 \times 10^{-9}(2 \sigma) .
\end{aligned}
$$

We consider a $3 \mathrm{GeV}$ theoretical uncertainty in computing $m_{h}$ as given above. Some of the reasons behind considering the spread are uncertainty in computing loop corrections up to three loops, top quark mass, renormalization scheme and scale dependence etc. [117-123].

We will now discuss the effect of introducing NH parameters within the mGMSB scenario and especially study the dependence of the SUSY spectra and observables of phenomenological interest on $\Lambda, M_{\text {mess }}$ and $\mu_{0}^{\prime}$. We remind that in this analysis the trilinear NH couplings are vanishing at the messenger scale whereas the higgsino mass soft-parameter $\mu_{0}^{\prime}$ is considered to arise from sources other than GMSB. This is following the discussion of section 2 .

\subsection{Effects on top-squarks and Higgs masses}

Figures 1(a) and 1(b) represent the scatter plots of Higgs boson mass with $\Lambda$ for $\tan \beta=10$ and 40 respectively when $M_{\text {mess }}$ and $\mu_{0}^{\prime}$ are scanned according to eq. (3.2). Here, the mass

\footnotetext{
${ }^{8}$ Henceforth we will refer $M_{\text {mess }}$ as the messenger scale.
} 


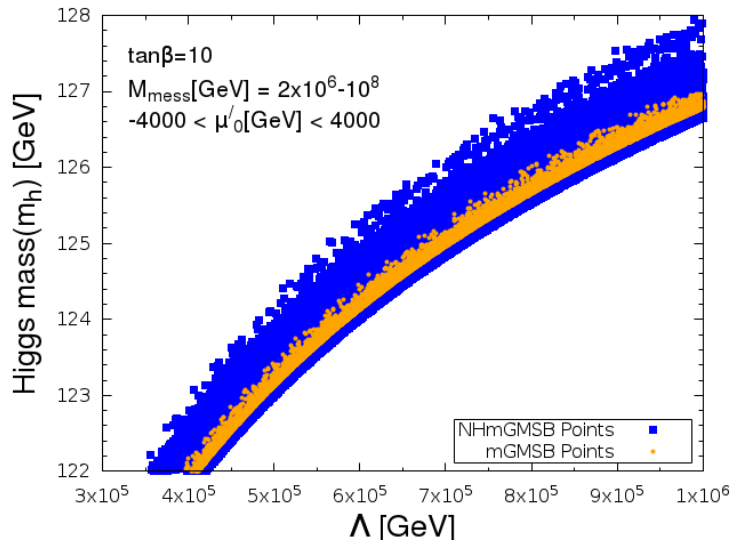

(a)

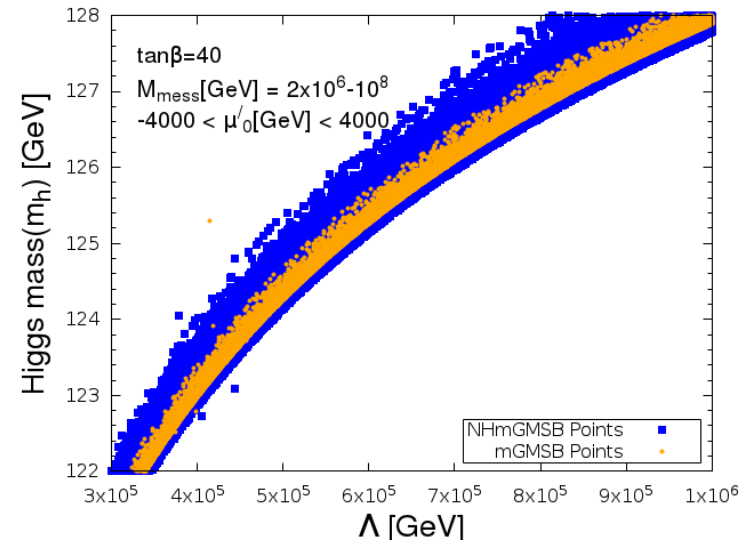

(b)

Figure 1. Scatter plots of $m_{h}$ with $\Lambda$ are shown for $\tan \beta=10$ and 40 when $M_{\text {mess }}$ and $\mu_{0}^{\prime}$ are scanned according to the ranges mentioned in eq. (3.2). The blue and yellow coloured regions correspond to NHmGMSB and mGMSB cases respectively.

of the lighter CP-even neutral Higgs boson $h$ which is standard-model like in its couplings satisfies the range mentioned in eq. (3.3). The yellow and blue zone refer to the results of mGMSB and NHmGMSB respectively. The lower limit of $\Lambda$ corresponding to the lower limit of $m_{h}$ (eq. (3.3)) for any given scenario either mGMSB or NHmGMSB decreases with an increase in $\tan \beta$. The spread of points for $m_{h}$ shown in blue (NHmGMSB) is quite large compared to the yellow region (mGMSB) for $\tan \beta=10$. However, this is not so for the case of $\tan \beta=40$. We note that $\mu+A_{t}^{\prime}$ appearing in the off-diagonal elements of the top-squark mass matrix is suppressed by $\tan \beta$. The larger top-squark mixing causes more prominent spread for low $\tan \beta$. Regarding $A_{t}$, it is seen that $A_{t}$ turns out to be negative. However, $X_{t}^{\prime}$ is not large enough to be in the maximal mixing zone characterized by $X_{t}^{\prime}=\sqrt{6} M_{S}$. This is also true for $X_{t}\left(=A_{t}-\mu \cot \beta\right)$ for the mGMSB case. Furthermore, it is found that $A_{t}^{\prime}$ at the EWSB scale approximately varies from $-550(-600) \mathrm{GeV}$ to $550(600) \mathrm{GeV}$ for $\tan \beta=10$ (40). $A_{t}^{\prime}$ comes with either sign because of its dependence via RGE on $\mu_{0}^{\prime}$, while the latter is scanned for both positive and negative values. Additionally, we note that $\mu_{0}^{\prime}$ may also contribute $[73,74,80]$ quite significantly to the value of $\mu$ which is obtained via REWSB. With all the above effects and estimates, $X_{t}^{\prime}$ is seen to be larger in the negative direction for $\tan \beta=10$ than $\tan \beta=40$. Because of a larger top-squark mixing, that results into a larger radiative corrections to the Higgs boson mass, the lowest value of $\Lambda$ satisfying the lower limit of $m_{h}$ for NHmGMSB is less than the same for mGMSB.

We will now explore the variation of Higgs boson mass $m_{h}$ with top-squark mass $m_{\tilde{t}_{1}}$ for $\tan \beta=10$ and 40 in figures 2(a) and figure 2(b) respectively, when $\Lambda, M_{\text {mess }}, \mu_{0}^{\prime}$ are scanned according to eq. (3.2). The colors carry the same convention as that of figure 1. For a given value of $m_{h}$, the lower limit of $m_{\tilde{t}_{1}}$ becomes smaller in NHmGMSB than that of mGMSB and this is more prominent for $\tan \beta=10$ because of a larger influence of $\left(\mu+A_{t}^{\prime}\right)$. For a given $m_{\tilde{t}_{1}}$, the amount of corrections in $m_{h}$ via $\mathrm{NH}$ terms may reach up to nearly $1 \mathrm{GeV}$ for $\tan \beta=10$ and $0.5 \mathrm{GeV}$ for $\tan \beta=40$. On the other hand, for a given $m_{h}$, the 


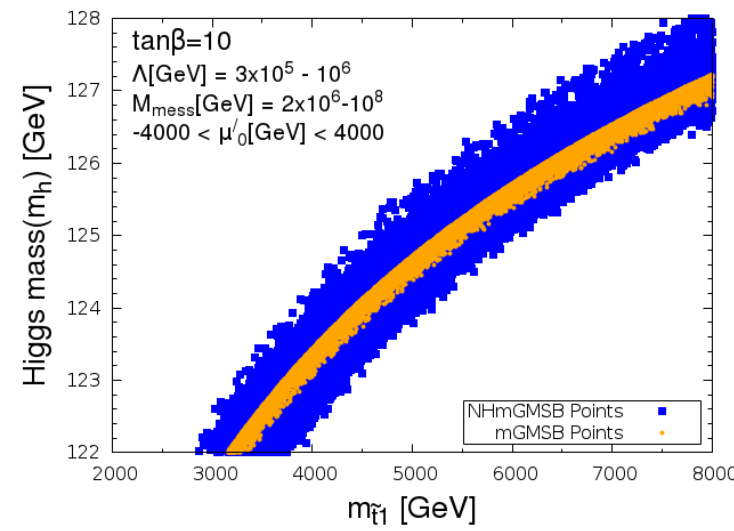

(a)

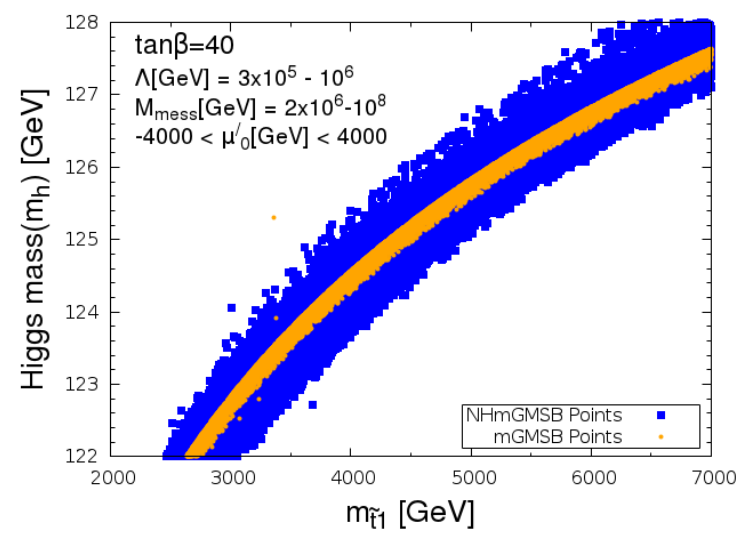

(b)

Figure 2. Scatter plots of $m_{h}$ with stop mass $m_{\tilde{t}_{1}}$ are shown for $\tan \beta=10$ and $40 . \Lambda, M_{\text {mess }}, \mu_{0}^{\prime}$ are scanned according to the ranges mentioned in eq. (3.2). The colors carry the same convention as that of figure 1 .

spread of $m_{\tilde{t}_{1}}$ is about $1 \mathrm{TeV}$ from its minimum to the maximum value for NHmGMSB within which the scattered points for mGMSB reside.

Figures $3(\mathrm{a})$ and $3(\mathrm{~b})$ show scatter plots of the lighter top-squark mass $m_{\tilde{t}_{1}}$ with $\Lambda$ corresponding to $\tan \beta=10$ and 40 respectively, where the Higgs boson mass $\left(m_{h}\right)$ satisfies the range of eq. (3.3). The blue and yellow zones refer to the results of NHmGMSB and mGMSB respectively. The Higgs boson mass lower limit is reached for a smaller value of $\Lambda$ for $\tan \beta=40$ in comparison with $\tan \beta=10$ for reasons described earlier. With the validity of the lower $\Lambda$ zone for a larger $\tan \beta$ via the $m_{h}$ lower limit, the top-squark mass $m_{\tilde{t}_{1}}$ finds its smallest value to be smaller for $\tan \beta=40$ compared to the same for $\tan \beta=10$. On the other hand, between $\tan \beta=10$ and 40 , the relative difference of the lowest value of $m_{\tilde{t}_{1}}$ between NHmGMSB and mGMSB is more enhanced for the lower value of $\tan \beta$ primarily because of a lesser degree of suppression of the contribution of $\mu+A_{t}^{\prime}$ in the top-squark mixing. The resulting reduction of $m_{\tilde{t}_{1}}$ in NHmGMSB (compared to mGMSB) is about 500 (200) GeV for $\tan \beta=10$ (40).

We will now explore the role of the messenger scale $M_{\text {mess }}$ in our analysis that would shed some light on the extent of RG evolutions of relevant parameters that have influence on the scalar masses including that of the Higgs boson as well as on the masses of the electroweakinos. The effects of evolutions are not likely to be as large as that occur in minimal supergravity (mSUGRA) [69] with much higher SUSY mediation scale, but as we have seen they may assist in lowering the top squark mass $m_{\tilde{t}_{1}}$ or enhancing $m_{h}$. Figure 4(a) and 4(b) show color contour plots in the ( $\Lambda$ vs $M_{\text {mess }}$ ) plane for NHmGMSB corresponding to $\tan \beta=10$ and 40 respectively when $\mu_{0}^{\prime}$ is scanned as in eq. (3.2). The side panels show the values of $m_{h}$. The figures show strong dependence of Higgs mass on $\Lambda$ (that sets the masses of the scalars as well as gauginos) and a weaker dependence on $M_{\text {mess }}$. However, we must mention that the upper limit of $M_{\text {mess }}$ is chosen relatively small in our analysis. This is based on our motivation to have the NH terms of being associated with a lesser 


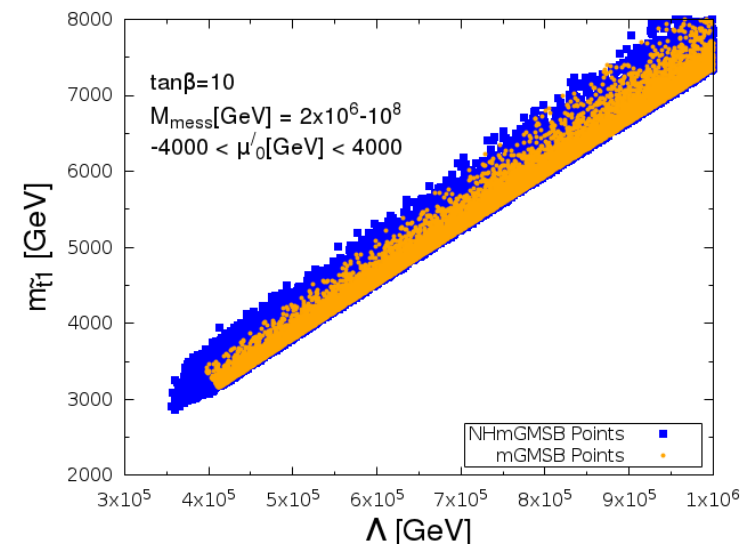

(a)

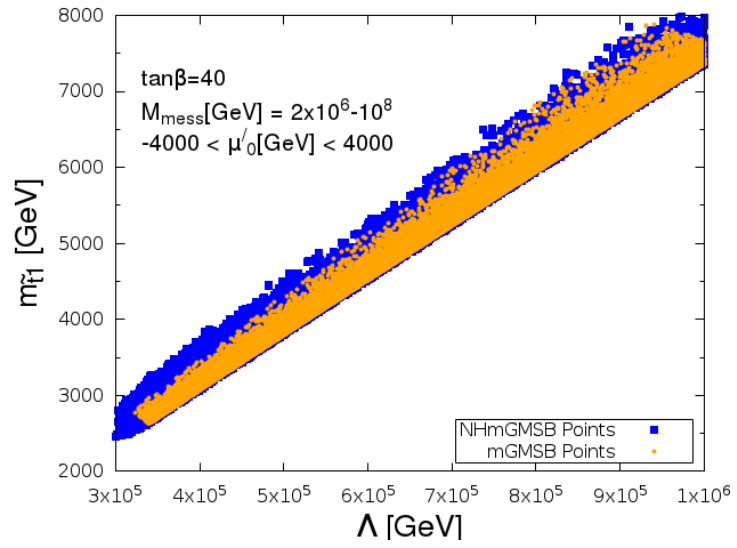

(b)

Figure 3. Scatter plots of the lighter top-squark mass $m_{\tilde{t}_{1}}$ with $\Lambda$ for $\tan \beta=10$ and 40 for the scanning ranges mentioned in eq. (3.2). The blue and yellow zones refer to the results of NHmGMSB and mGMSB respectively.

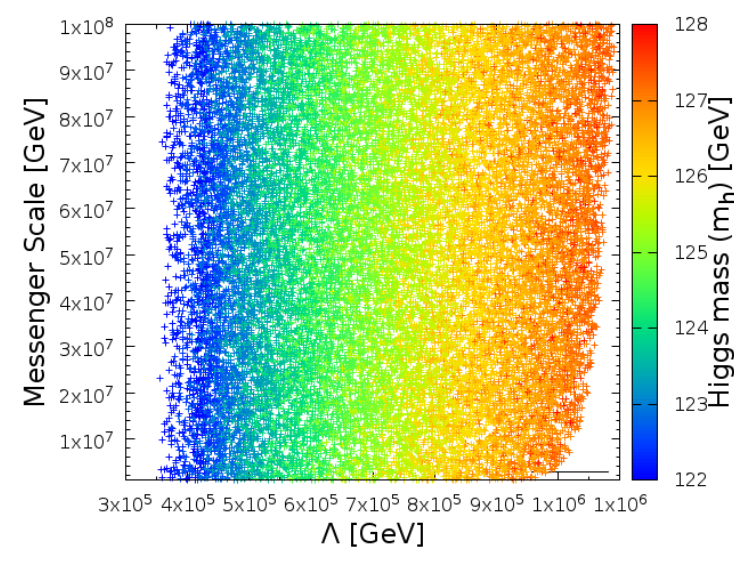

(a)

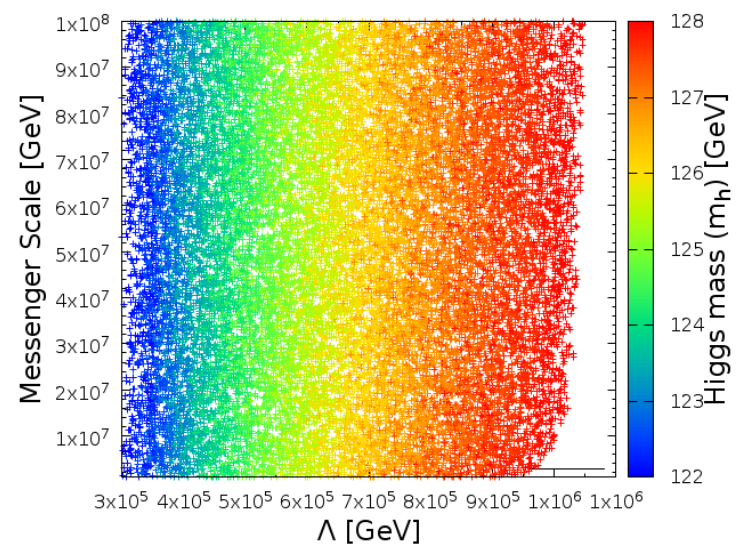

(b)

Figure 4. Scatter plots of $\Lambda$ against Messenger Scale $M_{\text {mess }}$ for $\tan \beta=10$ and 40 for NHmGMSB, corresponding to the scanning ranges mentioned in eq. (3.2). The side panels of each of the plots show the Higgs mass.

degree of mass suppression as mentioned in section 2, the reason of our working in a GMSB setup. A larger $M_{\text {mess }} \sim 10^{12} \mathrm{GeV}$ (not shown in this work) may increase both $A_{t}$ and $A_{t}^{\prime}$ significantly to higher values beyond what would be necessary to satisfy the Higgs boson mass limit for a reasonably chosen $\Lambda$.

It is important to note that there are a few beyond the mGMSB analyses [102, 124-128] for example those involving matter-messenger interactions in which the trilinear holomorphic soft terms may arise at one-loop level, thus becoming non-vanishing at the messenger scale. In such situations one obtains a significantly large amount of radiative corrections to $m_{h}$, a friendly feature to accommodate a Higgs boson as massive as $125 \mathrm{GeV}$ in models 
away from mGMSB. The NH trilinear terms will then similarly become non-vanishing at the above scale. Depending on the sign of $\mu_{0}^{\prime}$, this may give rise to a larger $A_{t}^{\prime}$ at the electroweak scale. The above in turn that may result into a smaller $m_{\tilde{t}_{1}}$ or positively contributing toward the radiative corrections to the Higgs boson mass $m_{h}$. Thus a smaller $\Lambda$ would be acceptable lowering the overall mass scale of sparticles. Additionally, as we will see below, the same consideration may significantly enhance the smuon mass mixing via a larger $A_{\mu}^{\prime}$. A large $A_{\mu}^{\prime}$ may effectively contribute to the SUSY contribution to muon $g-2$ significantly, because of an associated scaling by $\tan \beta[70]$.

\subsection{Phenomenological implications through the electroweakino sector}

As discussed in section 2 the electroweakino sectors are influenced by $\mu+\mu^{\prime}$ particularly when the combination that is close to the higgsino mass is lesser than the masses of relevant gauginos $M_{1}$ and $M_{2}$. We will discuss the phenomenological implications on $\operatorname{Br}(B \rightarrow$ $\left.X_{s}+\gamma\right)$ and muon $g-2$ in this context. The diagrams that are significant in MSSM for $\operatorname{Br}\left(B \rightarrow X_{s}+\gamma\right)$ are the loops involving $t-H^{ \pm}$and $\tilde{t}-\tilde{\chi}_{1}^{ \pm}$. Agreement of the SM result of $\operatorname{Br}\left(B \rightarrow X_{s}+\gamma\right)$ with the experimental data demands delicate cancellation between the contributions of the above loops. In $\operatorname{MSSM}, \operatorname{Br}\left(B \rightarrow X_{s}+\gamma\right)$ increases with $\tan \beta$ [65]. Additionally, there are Next to Leading Order (NLO) contributions from squark-gluino loops due to corrections of bottom and top Yukawa couplings, particularly for large values of $\tan \beta$. Here we will discuss the effects on $\operatorname{Br}\left(B \rightarrow X_{s}+\gamma\right)$ in relation to the results of ref. [70] that used unconstrained NH soft terms given at the weak scale (NHSSM). The $\tilde{t}-\widetilde{\chi}_{1}^{ \pm}$loop that is relevant for our study does not have an appreciable effect from top squark L-R mixing since both $A_{t}$ and $A_{t}^{\prime}$ that start from vanishing values at the messenger scale have only limited degree of evolutions. This is in contrast to the larger possible values assumed by the above trilinear parameters as analyzed in ref. [70]. Figure 5 shows the variation of $\operatorname{Br}\left(B \rightarrow X_{s}+\gamma\right)$ with $\mu_{0}^{\prime}$ in NHmGMSB for a given $\Lambda$ and $M_{\text {mess }}$. It shows some sharp increase in $\operatorname{Br}\left(B \rightarrow X_{s}+\gamma\right)$ for a given zone of $\mu_{0}^{\prime}$, particularly for a large $\tan \beta=40$. It is found that this is indeed the region when $\mu+\mu^{\prime}$ or the lighter chargino mass becomes small. The effect was also seen earlier [69, 79]. The corresponding mGMSB values of the same quantity are also shown where $m_{\tilde{\chi}_{1}^{ \pm}}$is not small. Apart from the cases with small $m_{\widetilde{\chi}_{1}^{ \pm}}, \operatorname{Br}\left(B \rightarrow X_{s}+\gamma\right)$ does not impose any serious constraint on NHmGMSB parameter space even for a large value $\tan \beta$ because of the smallness of $A_{t}$ and $A_{t}^{\prime}$ and large values of top-squark masses so as to satisfy the Higgs mass data. This is unlike NHSSM that recovers a large amount of parameter space discarded by $\operatorname{Br}\left(B \rightarrow X_{s}+\gamma\right)$ in MSSM for a large value of $\tan \beta[70]$.

We will now discuss the SUSY contributions to $(g-2)_{\mu}$, namely $a_{\mu}^{\text {SUSY }},{ }^{9}$ in the context of NHmGMSB against the results of the NHSSM analysis made in ref. [70]. At the oneloop level, $a_{\mu}^{\text {SUSY }}$ involves contributions from $\tilde{\chi}_{i}^{ \pm}-\tilde{\nu}_{\mu}$ and $\tilde{\chi}_{i}^{0}-\tilde{\mu}$ loops [129-134]. In the NHSSM analysis of ref. [70], because of a strong L-R mixing via $A_{\mu}^{\prime}$, it is the $\tilde{\chi}_{i}^{0}-\tilde{\mu}$ loop that contributes a significantly large amount to $a_{\mu}^{\mathrm{SUSY}}$. $A_{\mu}^{\prime}$ can be as small as $100 \mathrm{GeV}$ or even $50 \mathrm{GeV}$ to show very prominent effects. The largeness of the effect in NHSSM comes

$$
{ }^{9} a_{\mu}=\frac{1}{2}(g-2)_{\mu} .
$$




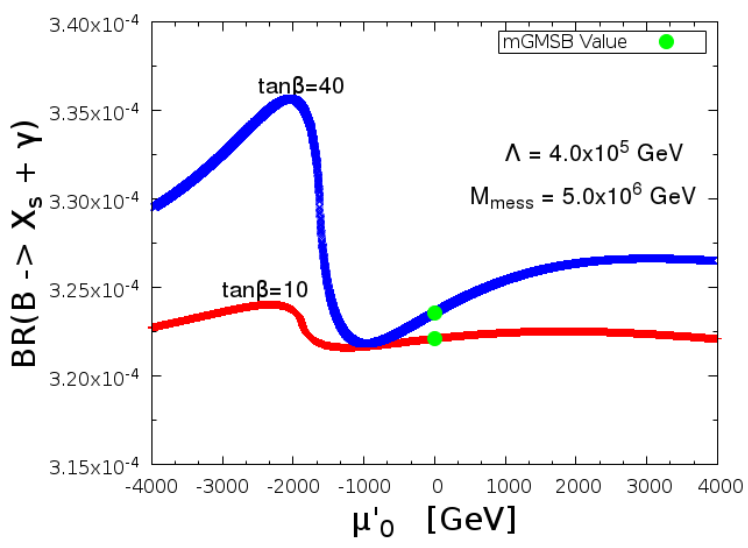

Figure 5. The variation of $\operatorname{Br}\left(B \rightarrow X_{s}+\gamma\right)$ with $\mu_{0}^{\prime}$ is shown for the shown values of $\Lambda$ and $M_{\text {mess }}$. The red and blue lines correspond to $\tan \beta=10$ and 40 respectively. Green filled circles on the top of the lines specify the corresponding mGMSB values.

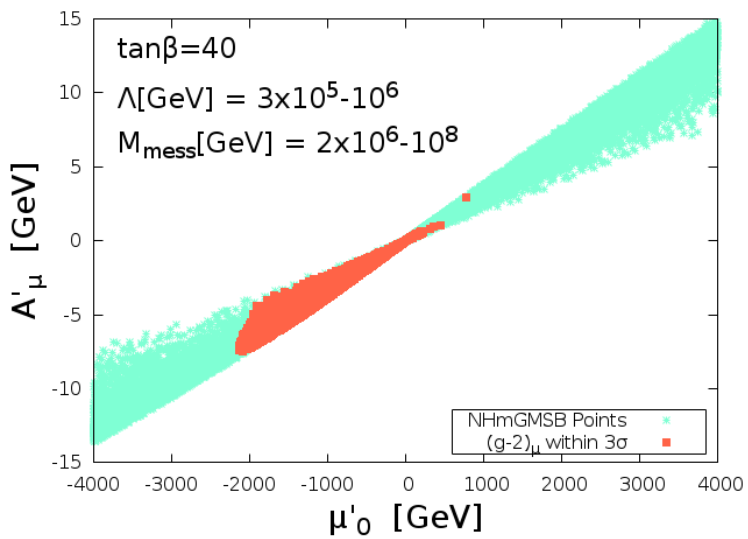

Figure 6. Scatter plot of $\mu_{0}^{\prime}$ vs $A_{\mu}^{\prime}$ where $\Lambda$ and $M_{\text {mess }}$ are varied according to the ranges mentioned in eqs. (3.2). The green points satisfy all the relevant constraints of eq. (3.3) while the orange points correspond to a region that satisfy the muon $g-2$ constraint at $3 \sigma$ level.

from an enhancement of $A_{\mu}^{\prime}$ via $\tan \beta$ and the diagram containing bino- $\tilde{\mu}_{L, R}$ in the loop that contributes to $a_{\mu}^{\mathrm{SUSY}}[135,136]$. In NHmGMSB, $A_{\mu}^{\prime}$ that starts from a vanishing value at $M_{\text {mess }}$ has only a limited degree of evolution [80] even with a large $\mu_{0}^{\prime}$. As a result, with an inadequate level of enhancement of $A_{\mu}^{\prime}$, we do not expect any large amount of contributions from the bino-smuon loops due to L-R mixing, unlike the NHSSM results of ref. [70]. Figure 6 shows a scatter plot of $\mu_{0}^{\prime}$ vs $A_{\mu}^{\prime}$ where $\Lambda$ and $M_{\text {mess }}$ are varied within the shown ranges of eq. (3.2). The green points are those that satisfy all the relevant constraints of eq. (3.3) while the orange points correspond to a region that satisfy the muon $g-2$ constraint at $3 \sigma$ level. The $3 \sigma$ region spreads more toward the negative zone of $\mu_{0}^{\prime}$. We should remind that $\mu$ is obtained via REWSB with a chosen positive sign, whereas $\mu_{0}^{\prime}$ assumes both the signs. Thus, a larger $a_{\mu}^{\mathrm{SUSY}}$ via a lighter higgsino mass can be obtained only in the negative direction of $\mu_{0}^{\prime}$. 


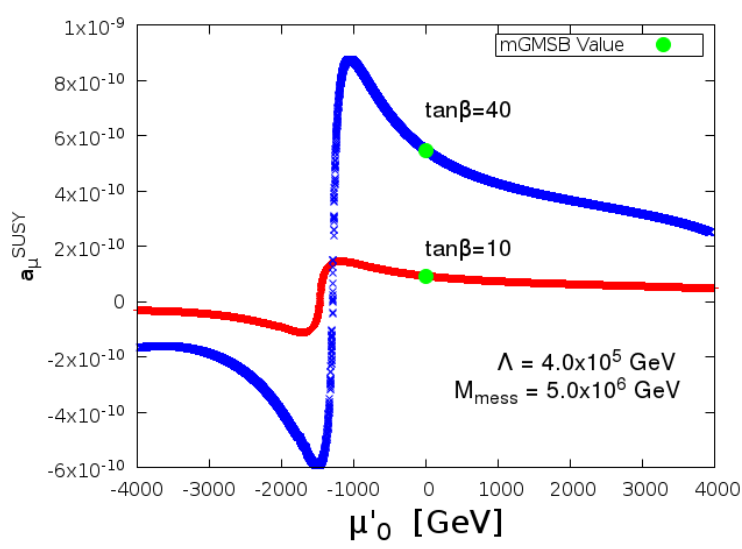

Figure 7. Plot of $a_{\mu}^{\text {SUSY }}$ vs $\mu_{0}^{\prime}$ for fixed $\Lambda$ and $M_{\text {mess }}$ for $\tan \beta=10$ and 40 . The corresponding mGMSB values of the same quantity are also shown by considering vanishing $\mu_{0}^{\prime}$.

The fact that the large $A_{\mu}^{\prime}$ regions are not associated with larger values of $a_{\mu}^{\mathrm{SUSY}}$, rather the region with smaller $\mu_{0}^{\prime}$ satisfy the $3 \sigma,{ }^{10}$ level of the constraint predominantly for a negative region of $\mu^{\prime}$ indicates the domination of charged and neutral higgsinos in the loop diagrams [135]. This is indeed displayed in figure 7 that shows a plot of $\mu_{0}^{\prime}$ vs $a_{\mu}^{\text {SUSY }}$ for a given $\Lambda$ and $M_{\text {mess }}$. The figure shows a sharp rise in $a_{\mu}^{\mathrm{SUSY}}$ for a small zone of $\mu_{0}^{\prime}$ that also increases with $\tan \beta$. It turns out that with the value of $\mu$ obtained from REWSB, and $\mu+\mu^{\prime}$ being the tree level higgsino mass, this rise corresponds to the light higgsino zone. ${ }^{11}$ The specific contributions to $a_{\mu}^{\text {SUSY }}$ arise from diagrams [135] like wino/higgsinosneutrino and wino/higgsino- $\tilde{\mu}_{L}$, bino/higgsino- $\tilde{\mu}_{L}$ or bino/higgsino- $\tilde{\mu}_{R}$. There may also be a significant degree of cancellations between the above diagrams. The $3 \sigma$ level of $a_{\mu}^{\text {SUSY }}$ region for $\mu_{0}^{\prime}$ as shown in figure 6 comes out to be around $-1500 \mathrm{GeV}$ to $200 \mathrm{GeV}$.

\subsection{Higgsino like NLSP decays}

The interaction Lagrangian of gravitino $\psi_{\mu}$ with other sparticles and SM particles is given by $\mathcal{L}=\sum_{\alpha=1}^{3} \mathcal{L}^{(\alpha)}$ where $\alpha$ stands for a given gauge group out of $\mathrm{SU}(3)_{C} \times \mathrm{SU}(2)_{L} \times \mathrm{U}(1)_{Y}$ and $\mathcal{L}^{(\alpha)}$ being given as [137],

$$
\mathcal{L}^{(\alpha)}=-\frac{i}{\sqrt{2} M_{p}}\left[\mathcal{D}_{\mu}^{(\alpha)} \phi^{* i} \bar{\psi}_{\nu} \gamma^{\mu} \gamma^{\nu} \chi_{L}^{i}-\mathcal{D}_{\mu}^{(\alpha)} \phi^{i} \bar{\chi}_{L}^{i} \gamma^{\nu} \gamma^{\mu} \psi_{\nu}\right]-\frac{i}{8 M_{p}} \bar{\psi}_{\mu}\left[\gamma^{\rho}, \gamma^{\sigma}\right] \gamma^{\mu} \lambda^{(\alpha) a} F_{\rho \sigma}^{(\alpha) a} .
$$

The covariant derivatives $\mathcal{D}_{\mu}^{(\alpha)}$ and $F_{\rho \sigma}^{(\alpha) a}$ are appropriately defined depending on the gauge group denoted by $\alpha$ and the generator index $a$ [137].

The resulting decay widths $\Gamma\left(\widetilde{\chi}_{1}^{0} \rightarrow \widetilde{G}+Z\right)$ and $\Gamma\left(\widetilde{\chi}_{1}^{0} \rightarrow \widetilde{G}+h\right)[94,98,138-140]$ are given as below. This is keeping in mind that $\widetilde{\chi}_{1}^{0}$, the NLSP candidate, is chosen to be higgsino dominated in nature and the decays into heavier Higgs bosons are kinematically disallowed. We also note that a higgsino type of NLSP couples only to the longitudinal $Z$

\footnotetext{
${ }^{10}$ We consider ref. [63] for the following: $a_{\mu}^{\mathrm{SM}}=11659182.3 \pm 4.3\left(\times 10^{-10}\right)$ and $a_{\mu}^{\exp }=11659209.1 \pm$ $6.3\left(\times 10^{-10}\right)$. This leads to a discrepancy of $26.8 \pm 7.6\left(\times 10^{-10}\right)(3.5 \sigma)$.

${ }^{11} \mu^{\prime}$ has a limited degree of evolution from $M_{\text {mess }}$ to the electroweak scale.
} 
component. In realistic scenarios with small amount of gaugino mixing in NLSP, which we will though ignore here, $\Gamma\left(\widetilde{\chi}_{1}^{0} \rightarrow \widetilde{G} \gamma\right)$ can be quite important. However, we will work only with an almost pure higgsino. For the above two decays we have,

$$
\begin{aligned}
& \Gamma\left(\widetilde{\chi}_{1}^{0} \rightarrow \widetilde{G} Z\right) \simeq \frac{m_{\widetilde{\chi}_{1}^{0}}^{5}}{96 \pi m_{\tilde{G}_{p}^{2}}^{2} M_{p}^{2}}\left|-N_{13} \cos \beta+N_{14} \sin \beta\right|^{2}\left(1-\frac{m_{Z}^{2}}{m_{\widetilde{\chi}_{1}^{0}}^{2}}\right)^{4}, \\
& \Gamma\left(\widetilde{\chi}_{1}^{0} \rightarrow \widetilde{G} h\right) \simeq \frac{m_{\widetilde{\chi}_{1}^{0}}^{5}}{96 \pi m_{\tilde{G}}^{2} M_{p}^{2}}\left|-N_{13} \sin \alpha+N_{14} \cos \alpha\right|^{2}\left(1-\frac{m_{h}^{2}}{m_{\widetilde{\chi}_{1}^{0}}^{2}}\right)^{4},
\end{aligned}
$$

where $N_{13}$ and $N_{14}$ are the higgsino related components of the LSP in the $\tilde{B}, \tilde{W}, \widetilde{H_{d}^{0}}, \widetilde{H_{u}^{0}}$ basis of the neutralino diagonalizing matrix $N_{i j}$ [15]. The gravitino mass is given by,

$$
m_{\tilde{G}}=\frac{\Lambda M_{\mathrm{mess}}}{\sqrt{3} M_{P}} .
$$

For our choice of parameter space $m_{\tilde{G}}$ is of order of few $\mathrm{keV}$. This allows us to neglect it in the expression of the phase space factor compared to other masses involved in the calculation of decay widths in eqs. (3.4), (3.5). The total decay width of a higgsino type of NLSP is given by,

$$
\Gamma^{\mathrm{tot}} \equiv \Gamma_{\mathrm{NLSP}}^{\mathrm{tot}} \simeq \Gamma\left(\widetilde{\chi}_{1}^{0} \rightarrow \widetilde{G} Z\right)+\Gamma\left(\widetilde{\chi}_{1}^{0} \rightarrow \widetilde{G} h\right)
$$

$\Gamma^{\text {tot }}$ would be strongly influenced by $\Lambda$ and $M_{\text {mess }}$ since $\Gamma^{\text {tot }} \propto \frac{1}{\left(\Lambda M_{\text {mess }}\right)^{2}} \cdot \Gamma^{\text {tot }}$ is also influenced by $\mu_{0}^{\prime}$ through the higgsino NLSP mass as well as the effect of higgsino mixing in $N_{i j}$ s. We explore the decay widths of eqs. (3.4) and (3.5) against the NLSP mass in figure 8 (a) and 8 (b) respectively. The fixed values of the chosen parameters are $\operatorname{sign}(\mu)=1$, $\tan \beta=35, \Lambda=4.5 \times 10^{5} \mathrm{GeV}$ and $M_{\text {mess }}=8 \times 10^{7} \mathrm{GeV} . \quad \mu_{0}^{\prime}$ is scanned over a range $-4 \mathrm{TeV}$ to $4 \mathrm{TeV}$. Both the decay widths increase with NLSP mass as long as the NLSP is substantially a higgsino. This is so until $m_{\widetilde{\chi}_{1}^{0}}$ is about $550 \mathrm{GeV}$. Once, the NLSP is found to be an admixture of a higgsino and bino or essentially a bino each of the decay widths is bound to decrease rapidly. This happens after it attains a peak similar to what is apparent in figure $8(\mathrm{a})$ or $8(\mathrm{~b})$. In short of other relevant decay modes, eq. (3.6) would then no longer be valid. Regarding the composition of the NLSP and its effect on $\Gamma\left(\widetilde{\chi}_{1}^{0} \rightarrow \widetilde{G} Z\right)$ and $\Gamma\left(\widetilde{\chi}_{1}^{0} \rightarrow \widetilde{G} h\right)$ we would like to point out that both $N_{13}$ and $N_{14}$ change signs over the scanned region of parameter space. However, $N_{14}$ plays a dominant role for both types of decays unless $\tan \beta$ is very small. In eq. (3.4) the $N_{13}$ contribution is very subdominant since $\cos \beta$ is small. A similar subdominant role for $N_{13}$ is also true in eq. (3.5) since we have a decoupling Higgs boson scenario where $\sin \alpha$ becomes small [20]. The above causes both $\Gamma\left(\widetilde{\chi}_{1}^{0} \rightarrow \widetilde{G} Z\right)$ and $\Gamma\left(\widetilde{\chi}_{1}^{0} \rightarrow \widetilde{G} h\right)$ to peak at similar values of the NLSP mass. Apart from the above, because of the possibility of both the signs for $N_{13}$ and $N_{14}$, figures 8 (a) and 8 (b) show that each of the decay widths has two branches or in other words is a double-valued function of $m_{\widetilde{\chi}_{1}^{0}}$ depending on the two cases $\mu>\left|\mu^{\prime}\right|$ and $\mu<\left|\mu^{\prime}\right|$. The heights of the decay widths of the two branches for each of $\Gamma\left(\widetilde{\chi}_{1}^{0} \rightarrow \widetilde{G} Z\right)$ and $\Gamma\left(\widetilde{\chi}_{1}^{0} \rightarrow \widetilde{G} h\right)$ differ because of a varying degree of radiative corrections to the NLSP mass. Considering 


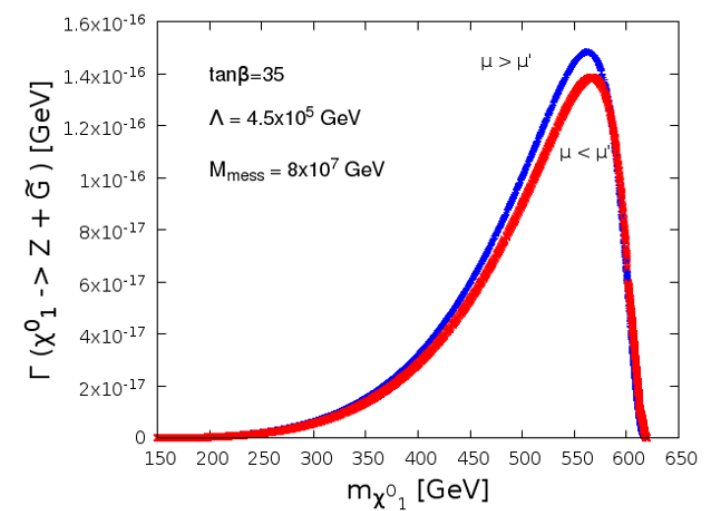

(a)

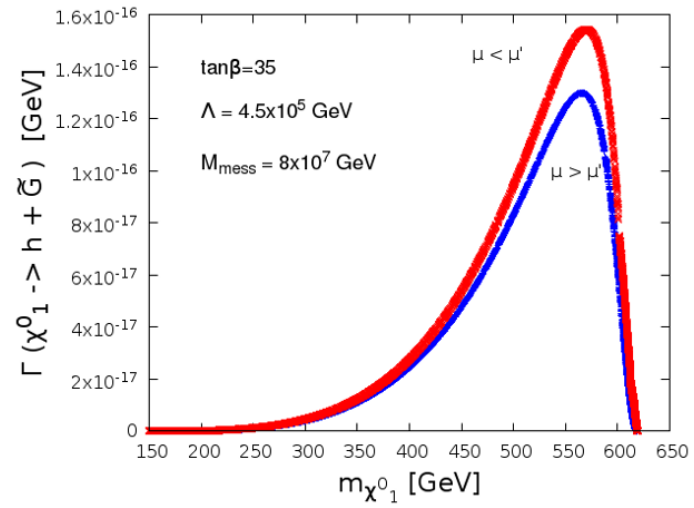

(b)

Figure 8. Plot of decay width vs. NLSP mass for $\widetilde{\chi}_{1}^{0} \rightarrow \widetilde{G}+Z$ and $\widetilde{\chi}_{1}^{0} \rightarrow \widetilde{G}+h$ channel for $\mu>0$ when $\mu^{\prime}$ is scanned over a range (eq. (3.2)) to probe the higgsino NLSP zone. The blue and red points refer to $\mu>\left|\mu^{\prime}\right|$ and $\mu<\left|\mu^{\prime}\right|$ respectively.

$m_{\widetilde{\chi}_{1}^{0}} \simeq 575 \mathrm{GeV}$ (near the peaks) the approximate sum of the two decay widths is around $2.8 \times 10^{-16} \mathrm{GeV}$ for the given parameter point. This leads to $1 / \Gamma^{\text {tot }} \simeq 2 \times 10^{-9} \mathrm{sec} \simeq 70 \mathrm{~cm}$. In general, the mean decay length of $\chi_{1}^{0}$ as NLSP with energy $E$ in the laboratory frame is given by [94, 139, 140],

$$
d \simeq\left(E^{2} / m_{\chi_{1}^{0}}^{2}-1\right)^{1 / 2} / \Gamma^{\text {tot }}
$$

Figure 9 (a) shows a scatter plot of decay width $\Gamma^{\text {tot }}$ vs. $m_{\widetilde{\chi}_{1}^{0}}$ for a higgsino dominated NLSP over the scanned parameter region of eq. (3.2). The higgsino fraction is shown in graded color with a reference color bar on the right. Only highly higgsino dominated NLSP region is considered. Figure $9(\mathrm{~b})$ shows a similar scatter plot in the plane of $\Gamma^{\text {tot }}$ vs. $F\left(=\Lambda M_{\text {mess }}\right)$ where the NLSP mass is shown in a graded color with a reference color bar on the right. The range of variation of $\Gamma^{\text {tot }}$ is from $10^{-22}$ to $10^{-12} \mathrm{GeV}$ implying $1 / \Gamma^{\text {tot }}$ to be within $\simeq 10^{-3}$ sec to $\simeq 10^{-13}$ sec or $\simeq 1000 \mathrm{~km}$ to $0.1 \mathrm{~mm}$ respectively. The decay lengths when computed in the laboratory frame would point out a long range of values indicating decays occurring both within and outside the detector. Collider studies of probing the higgsino NLSP decays for suitable values of $\Lambda M_{\text {mess }}$ may be performed similar to the analyses made in refs. [141-145]. This is however beyond the scope of the present work.

Finally, we present two representative points A and B in table 1 for the spectra of NHmGMSB to demonstrate the degree of evolution of the parameters connected with the $\mathrm{NH}$ terms while choosing the lighter chargino and the NLSP to be higgsino dominated in nature. This is generally unavailable in mGMSB where the NLSP as the lightest neutralino it is typically bino dominated in its composition. The spectra is generally heavy because of the requirement of satisfying the Higgs boson mass limit. We remind that the effect of the NH terms can be increased significantly and the spectra may be lighter while that would also satisfy the muon $g-2$ data, if we go beyond the mGMSB setup. This may 


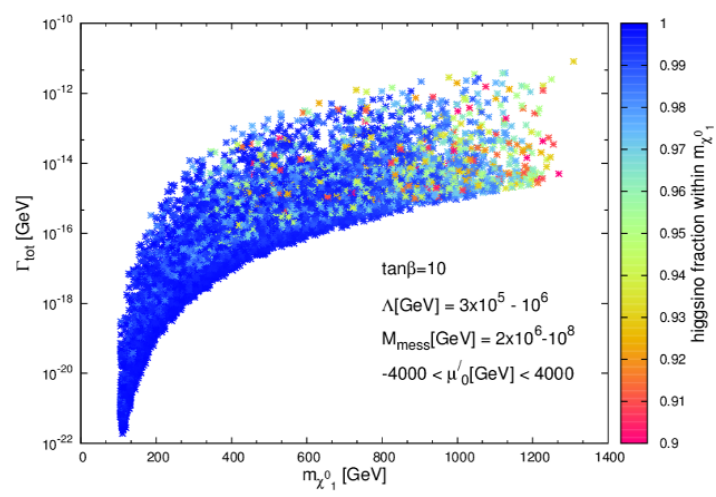

(a)

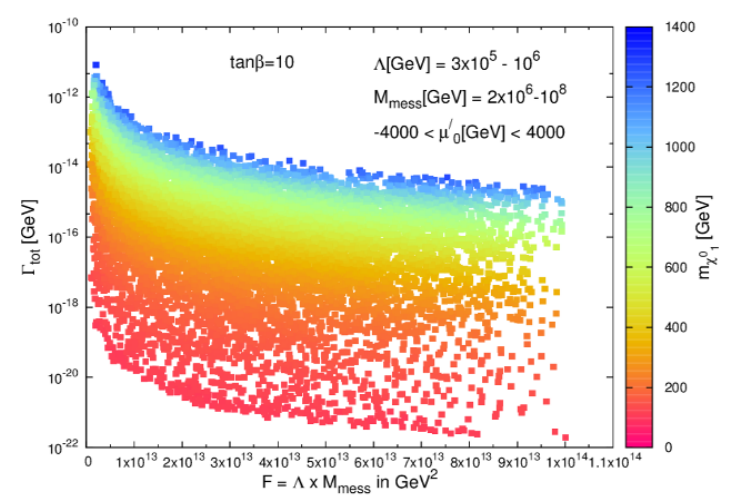

(b)

Figure 9. (a) Scatter plot of decay width $\Gamma^{\text {tot }}$ vs. $m_{\widetilde{\chi}_{1}^{0}}$ for a higgsino dominated NLSP over the scanned parameter region of eq. (3.2). The higgsino fraction is shown in graded color with a reference color bar on the right. (b) Similar scatter plot in the plane of $\Gamma^{\text {tot }}$ vs. $F$ where the NLSP mass is shown in a graded color with a reference color bar on the right.

be possible if the trilinear couplings for $\mathrm{NH}$ terms may have non-vanishing values at the messenger scale.

\section{Conclusion}

It was seen that SUSY models may include non-holomorphic terms like $\phi^{2} \phi^{*}$ and $\psi \psi$ that can be characterized as soft SUSY breaking in nature in the absence of a gauge singlet field. The broad applicability of the terms in various possible SUSY models makes such inclusion very important. In particular, the above terms may relax stringency to accommodate various phenomenological data in MSSM. These would be additional interactions than the usual soft SUSY breaking terms like that for the scalar masses (non-holomorphic) and trilinear and bilinear interactions that are holomorphic in nature. There have been studies on non-holomorphic MSSM referred as NHSSM that included such terms. The specific areas of impact that NHSSM makes are in the phenomenologies involving i) L-R mixing of squarks or sleptons via trilinear non-holomorphic terms containing the conjugate higgs fields, ii) higgsino mass soft SUSY breaking non-holomorphic term that results into higgsino components of electroweakinos to have parts coming from both superpotential as well as soft breaking origins and iii) the tree level electroweak fine-tuning to have no essential correlation with the higgsino mass, unlike MSSM. The authors of refs. [70, 82] performed phenomenological MSSM (pMSSM) type of analysis where all the soft parameters including the NH ones are provided at the weak scale. These were away from previous works that used mostly CMSSM inspired setup where in addition to the usual CMSSM inputs corresponding to the gauge coupling unification scale, the $\mathrm{NH}$ soft parameters were either given a) at the unification scale or b) at the electroweak scale. It was however shown that in the absence of a gauge singlet superfield, a hidden sector based F-type SUSY breaking scenario with two chiral superfields would lead to such NH soft terms [71]. The terms arise out of D-term contributions. On the other hand, supergravity scenarios with high scale SUSY breaking 


\begin{tabular}{|c|c|c|}
\hline Parameters & A & B \\
\hline$\Lambda$ & $3.65 \times 10^{5}$ & $3.16 \times 10^{5}$ \\
\hline$M_{\text {mess }}$ & $9.742 \times 10^{6}$ & $8.073 \times 10^{6}$ \\
\hline $\tan \beta$ & 10 & 40 \\
\hline$A_{0}^{\prime}$ & 0 & 0 \\
\hline$\mu_{0}^{\prime}$ & -1898 & -1144 \\
\hline$A_{t}$ & -787 & -686 \\
\hline$A_{b}$ & -136 & -430 \\
\hline$A_{\tau}$ & -14 & -38 \\
\hline$A_{t}^{\prime}$ & -210 & -147 \\
\hline$A_{b}^{\prime}$ & -55 & -121 \\
\hline$A_{\tau}^{\prime}$ & -23 & -57 \\
\hline$A_{\mu}^{\prime}$ & -1.4 & -3.4 \\
\hline$m_{h}$ & 122.1 & 122.3 \\
\hline$m_{H}, m_{H^{ \pm}}, m_{A}$ & $2047,2047,2047$ & $1425,1425,1425$ \\
\hline$m_{\tilde{t}_{1,2}}$ & 3090,3458 & 2651,2949 \\
\hline$m_{\tilde{b}_{1,2}}$ & 3357,3453 & 2841,2946 \\
\hline$m_{\tilde{\tau}_{1,2}}$ & 695,1315 & 566,594 \\
\hline$m_{\tilde{\chi}_{1,2}^{0}}$ & 432,451 & 202,212 \\
\hline$m_{\tilde{\chi}_{1,2}^{ \pm}}$ & 446,981 & 210,846 \\
\hline$m_{\tilde{g}}$ & 2636 & 2311 \\
\hline NLSP Composition & $\tilde{\chi}_{1}^{0} \approx 86 \% \tilde{H}$ like & $\chi_{1}^{0} \approx 98 \% \tilde{H}$ like \\
\hline$B R\left(B \rightarrow X_{s}+\gamma\right)$ & $3.22 \times 10^{-4}$ & $3.21 \times 10^{-4}$ \\
\hline$B R\left(B_{s} \rightarrow \mu^{+} \mu^{-}\right)$ & $3.27 \times 10^{-9}$ & $3.28 \times 10^{-9}$ \\
\hline$a_{\mu}^{S U S Y}$ & $1.027 \times 10^{-10}$ & $7.88 \times 10^{-10}$ \\
\hline
\end{tabular}

Table 1. Representative Points for NHmGMSB: all the dimensional parameters are in GeV.

could include such NH terms with mass scale suppression nearing the Planck scale [71]. Irrespective of the above, there have been analyses that worked out the phenomenologies of including such soft terms in a CMSSM setup or extensions like non-universal gaugino or non-universal Higgs scalar scenarios.

In this work, we first investigate the NH soft SUSY breaking models in a low scale SUSY breaking context. We choose to perform the analysis within a backbone provided by the minimal Gauge mediated SUSY Breaking (mGMSB) framework. We however assume that the higgsino mixing soft term parameter $\mu^{\prime}$ to have a SUSY breaking origin away from mGMSB. Thus, by having an entirely independent SUSY breaking origin for 
the higgsino mass soft term we essentially overcome any issue of the reparametrization invariance involving unrelated quantities as mentioned in the text. We focus on the degree of influence of the NH terms in an mGMSB setup (NHmGMSB) in comparison with the NHSSM work mentioned earlier. We note that the NH terms may affect various SUSY parameters including the Higgs scalar mass parameters through the RGEs. Thus, $\mu$, as obtained via REWSB may be non-negligibly modified once the NH terms are included.

We show that the level of the L-R mixing of top-squarks can potentially reduce the $m_{\tilde{t}_{1}}$ to a reasonably large extent, in turn increasing the radiative corrections to Higgs boson mass. Thus, a smaller than required $\left|A_{t}\right|$ value can do the job of satisfying the lower bound of $m_{h}$ compared to the mGMSB case since a compensating contribution may come from appropriate NH couplings. Of course, the enhancement of radiative corrections to the higgs boson mass is lesser when we compare our result with that of NHSSM. This is simply due to the limited degree of RG evolution of $A_{t}$ and $A_{t}^{\prime}$ from $M_{\text {mess }}$ down to the electroweak scale starting from their vanishing values at the higher scale. Regarding the effects on the electroweakino sector, we observe that the high level of enhancement (via a factor of $\tan \beta$ ) of the SUSY contributions to muon $g-2$ as that happens in NHSSM via $A_{\mu}^{\prime}$ through the L-R mixing contributions of smuons is absent in NHmGMSB. This is because $A_{\mu}^{\prime}$ does not become sufficiently large at the weak scale. The smallness of $A_{\mu}^{\prime}$ can, however, be avoided in non-minimal GMSB cases where the NH trilinear coupling $A_{0}^{\prime}$ need not be vanishing at the messenger scale. Simultaneously, the higgs boson mass $m_{h}$ would receive a larger amount of radiative correction through enhanced $A_{t}^{\prime}$. A larger $a_{\mu}^{\mathrm{SUSY}}$ comes from the limited zone of light higgsino mass in NHmGMSB. The same light higgsino mass zone may enhance the $\operatorname{Br}\left(B \rightarrow X_{s}+\gamma\right)$ contribution via the chargino loops. However, the constraint from $\operatorname{Br}\left(B \rightarrow X_{s}+\gamma\right)$ is well satisfied at the $2 \sigma$ level over the relevant parameter space of NHmGMSB simply because of generally large top-squark masses. This in turn arises from the requirement of satisfying the Higgs mass bound. We further estimate the decay of the lightest neutralino as NLSP into gravitino and $Z$-boson or $h$-boson while considering only a higgsino dominated NLSP. This is in contrast to a typical mGMSB scenario where $\widetilde{\chi}_{1}^{0}$ is bino-dominated in its composition. We probed the entire parameter space of NHmGMSB to find a large degree of variation of decay lifetimes that may correspond to a decay length of less than a millimeter to hundreds of kilometer in the rest frame of the NLSP. Relevant collider analyses may be made by finding the length in the laboratory frame of the detector. Depending on whether the NLSP decay is happening within or outside the detector one can probe NHmGMSB for a higgsino type of NLSP. Finally, we have presented two representative points for the spectra of NHmGMSB to demonstrate the extent of RG evolutions parameters related to the NH soft terms. The two spectra are on the heavier side so as to accommodate the Higgs mass data. However, a non-minimal GMSB scenario that allows having non-vanishing trilinear parameters $A_{0}$ and $A_{0}^{\prime}$ at the messenger scale would show a very significant effect on the scalar sector including the Higgs mass apart from potentially providing an enhanced $a_{\mu}^{\mathrm{SUSY}}$ via a large L-R mixing of the smuons. This is beyond the focus of the present analysis. Finally, considering the generic nature of the NH soft terms, it is important to explore their effect in varying SUSY breaking scenarios and scales. It can also be significant in respect of global analyses of various SUSY models. 


\section{Acknowledgments}

UC would like to thank Anirban Kundu and Sourov Roy for a brief discussion. UC and SM are grateful to Abhishek Dey for many helpful suggestions.

Open Access. This article is distributed under the terms of the Creative Commons Attribution License (CC-BY 4.0), which permits any use, distribution and reproduction in any medium, provided the original author(s) and source are credited.

\section{References}

[1] ATLAS, CMS collaborations, Combined Measurement of the Higgs Boson Mass in pp Collisions at $\sqrt{s}=7$ and $8 \mathrm{TeV}$ with the ATLAS and CMS Experiments, Phys. Rev. Lett. 114 (2015) 191803 [arXiv: 1503.07589] [INSPIRE].

[2] ATLAS collaboration, Observation of a new particle in the search for the Standard Model Higgs boson with the ATLAS detector at the LHC, Phys. Lett. B 716 (2012) 1 [arXiv:1207.7214] [INSPIRE].

[3] CMS collaboration, Observation of a new boson at a mass of $125 \mathrm{GeV}$ with the CMS experiment at the LHC, Phys. Lett. B 716 (2012) 30 [arXiv:1207.7235] [INSPIRE].

[4] https://twiki.cern.ch/twiki/bin/view/AtlasPublic/SupersymmetryPublicResults.

[5] https://twiki.cern.ch/twiki/bin/view/CMSPublic/PhysicsResultsSUS.

[6] A.H. Chamseddine, R.L. Arnowitt and P. Nath, Locally Supersymmetric Grand Unification, Phys. Rev. Lett. 49 (1982) 970 [INSPIRE].

[7] R. Barbieri, S. Ferrara and C.A. Savoy, Gauge Models with Spontaneously Broken Local Supersymmetry, Phys. Lett. 119B (1982) 343 [INSPIRE].

[8] L.J. Hall, J.D. Lykken and S. Weinberg, Supergravity as the Messenger of Supersymmetry Breaking, Phys. Rev. D 27 (1983) 2359 [INSPIRE].

[9] P. Nath, R.L. Arnowitt and A.H. Chamseddine, Gauge Hierarchy in Supergravity Guts, Nucl. Phys. B 227 (1983) 121 [INSPIRE].

[10] N. Ohta, Grand unified theories based on local supersymmetry, Prog. Theor. Phys. 70 (1983) 542 [INSPIRE].

[11] P. Nath, R. Arnowitt and A.H. Chamseddine, Applied N=1 Supergravity, World Scientific, Singapore (1984).

[12] H.P. Nilles, Supersymmetry, supergravity and particle physics, Phys. Rept. 110 (1984) 1.

[13] J.D. Lykken, Introduction to supersymmetry, hep-th/9612114 [INSPIRE].

[14] J. Wess and J. Bagger, Supersymmetry and Supergravity, second edition, Princeton (1991).

[15] M. Drees, P. Roy and R.M. Godbole, Theory and Phenomenology of Sparticles, World Scientific, Singapore (2005).

[16] H. Baer and X. Tata, Weak scale supersymmetry: From superfields to scattering events, Cambridge University Press, Cambridge U.K. (2006).

[17] D.J.H. Chung, L.L. Everett, G.L. Kane, S.F. King, J.D. Lykken and L.-T. Wang, The Soft supersymmetry breaking Lagrangian: Theory and applications, Phys. Rept. 407 (2005) 1 [hep-ph/0312378] [INSPIRE]. 
[18] H.E. Haber and G. Kane, The search for supersymmetry: probing physics beyond the standard model, Phys. Rept. 117 (1985) 75.

[19] S.P. Martin, A Supersymmetry primer, hep-ph/9709356 [INSPIRE].

[20] A. Djouadi, The Anatomy of electro-weak symmetry breaking. II. The Higgs bosons in the minimal supersymmetric model, Phys. Rept. 459 (2008) 1 [hep-ph/0503173] [INSPIRE].

[21] R. Barbieri and G.F. Giudice, Upper Bounds on Supersymmetric Particle Masses, Nucl. Phys. B 306 (1988) 63 [INSPIRE].

[22] J.R. Ellis, K. Enqvist, D.V. Nanopoulos and F. Zwirner, Observables in Low-Energy Superstring Models, Mod. Phys. Lett. A 1 (1986) 57 [InSPIRE].

[23] R. Kitano and Y. Nomura, A Solution to the supersymmetric fine-tuning problem within the MSSM, Phys. Lett. B 631 (2005) 58 [hep-ph/0509039] [INSPIRE].

[24] M.E. Cabrera, J.A. Casas and R. Ruiz de Austri, Bayesian approach and Naturalness in MSSM analyses for the LHC, JHEP 03 (2009) 075 [arXiv:0812.0536] [INSPIRE].

[25] D.M. Ghilencea, A new approach to Naturalness in SUSY models, PoS(Corfu2012) 034 [arXiv:1304.1193] [INSPIRE].

[26] D.M. Ghilencea, Fixing the EW scale in supersymmetric models after the Higgs discovery, Nucl. Phys. B 876 (2013) 16 [arXiv:1302.5262] [InSPIRE].

[27] D.M. Ghilencea and G.G. Ross, The fine-tuning cost of the likelihood in SUSY models, Nucl. Phys. B 868 (2013) 65 [arXiv: 1208.0837] [InSPIRE].

[28] H. Baer, V. Barger, P. Huang, A. Mustafayev and X. Tata, Radiative natural SUSY with a 125 GeV Higgs boson, Phys. Rev. Lett. 109 (2012) 161802 [arXiv: 1207.3343] [INSPIRE].

[29] H. Baer, V. Barger and D. Mickelson, How conventional measures overestimate electroweak fine-tuning in supersymmetric theory, Phys. Rev. D 88 (2013) 095013 [arXiv:1309.2984] [INSPIRE].

[30] M.W. Cahill-Rowley, J.L. Hewett, A. Ismail and T.G. Rizzo, The Higgs Sector and Fine-Tuning in the pMSSM, Phys. Rev. D 86 (2012) 075015 [arXiv:1206.5800] [INSPIRE].

[31] M.W. Cahill-Rowley, J.L. Hewett, A. Ismail and T.G. Rizzo, More energy, more searches, but the phenomenological MSSM lives on, Phys. Rev. D 88 (2013) 035002 [arXiv:1211.1981] [INSPIRE].

[32] M. Cahill-Rowley, R. Cotta, A. Drlica-Wagner, S. Funk, J. Hewett, A. Ismail et al., Complementarity of dark matter searches in the phenomenological MSSM, Phys. Rev. D 91 (2015) 055011 [arXiv: 1405.6716] [INSPIRE].

[33] M. Perelstein and C. Spethmann, A Collider signature of the supersymmetric golden region, JHEP 04 (2007) 070 [hep-ph/0702038] [INSPIRE].

[34] C. Boehm, P.S.B. Dev, A. Mazumdar and E. Pukartas, Naturalness of Light Neutralino Dark Matter in pMSSM after LHC, XENON100 and Planck Data, JHEP 06 (2013) 113 [arXiv:1303.5386] [INSPIRE].

[35] K.L. Chan, U. Chattopadhyay and P. Nath, Naturalness, weak scale supersymmetry and the prospect for the observation of supersymmetry at the Tevatron and at the CERN LHC, Phys. Rev. D 58 (1998) 096004 [hep-ph/9710473] [INSPIRE].

[36] G. Jungman, M. Kamionkowski and K. Griest, Supersymmetric dark matter, Phys. Rept. 267 (1996) 195 [hep-ph/9506380] [INSPIRE]. 
[37] G. Bertone, D. Hooper and J. Silk, Particle dark matter: Evidence, candidates and constraints, Phys. Rept. 405 (2005) 279 [hep-ph/0404175] [INSPIRE].

[38] Planck collaboration, P.A.R. Ade et al., Planck 2015 results. XIII. Cosmological parameters, Astron. Astrophys. 594 (2016) A13 [arXiv:1502.01589] [INSPIRE].

[39] S. Bhattacharya, U. Chattopadhyay, D. Choudhury, D. Das and B. Mukhopadhyaya, Non-universal scalar mass scenario with Higgs funnel region of SUSY dark matter: A Signal-based analysis for the Large Hadron Collider, Phys. Rev. D 81 (2010) 075009 [arXiv: 0907.3428] [INSPIRE].

[40] U. Chattopadhyay, D. Das and D.P. Roy, Mixed Neutralino Dark Matter in Nonuniversal Gaugino Mass Models, Phys. Rev. D 79 (2009) 095013 [arXiv: 0902.4568] [InSPIRE].

[41] U. Chattopadhyay, D. Das, D.K. Ghosh and M. Maity, Probing the light Higgs pole resonance annihilation of dark matter in the light of XENON100 and CDMS-II observations, Phys. Rev. D 82 (2010) 075013 [arXiv:1006.3045] [INSPIRE].

[42] U. Chattopadhyay and D. Das, Higgs funnel region of SUSY dark matter for small tan beta, $R G$ effects on pseudoscalar Higgs boson with scalar mass non-universality, Phys. Rev. D 79 (2009) 035007 [arXiv:0809.4065] [INSPIRE].

[43] U. Chattopadhyay, A. Corsetti and P. Nath, WMAP constraints, SUSY dark matter and implications for the direct detection of SUSY, Phys. Rev. D 68 (2003) 035005 [hep-ph/0303201] [INSPIRE].

[44] S. Akula, M. Liu, P. Nath and G. Peim, Naturalness, Supersymmetry and Implications for LHC and Dark Matter, Phys. Lett. B 709 (2012) 192 [arXiv:1111.4589] [INSPIRE].

[45] J.L. Feng, K.T. Matchev and T. Moroi, Focus points and naturalness in supersymmetry, Phys. Rev. D 61 (2000) 075005 [hep-ph/9909334] [inSPIRE].

[46] J.L. Feng, K.T. Matchev and T. Moroi, Multi - TeV scalars are natural in minimal supergravity, Phys. Rev. Lett. 84 (2000) 2322 [hep-ph/9908309] [INSPIRE].

[47] J.L. Feng, K.T. Matchev and F. Wilczek, Neutralino dark matter in focus point supersymmetry, Phys. Lett. B 482 (2000) 388 [hep-ph/0004043] [INSPIRE].

[48] U. Chattopadhyay, A. Datta, A. Datta, A. Datta and D.P. Roy, LHC signature of the minimal SUGRA model with a large soft scalar mass, Phys. Lett. B 493 (2000) 127 [hep-ph/0008228] [INSPIRE].

[49] U. Chattopadhyay, T. Ibrahim and D.P. Roy, Electron and neutron electric dipole moments in the focus point scenario of SUGRA model, Phys. Rev. D 64 (2001) 013004 [hep-ph/0012337] [INSPIRE].

[50] J.L. Feng and F. Wilczek, Advantages and distinguishing features of focus point supersymmetry, Phys. Lett. B 631 (2005) 170 [hep-ph/0507032] [INSPIRE].

[51] S.P. Das, A. Datta, M. Guchait, M. Maity and S. Mukherjee, Focus Point SUSY at the LHC Revisited, Eur. Phys. J. C 54 (2008) 645 [arXiv:0708.2048] [InSPIRE].

[52] P. Draper, J.L. Feng, P. Kant, S. Profumo and D. Sanford, Dark Matter Detection in Focus Point Supersymmetry, Phys. Rev. D 88 (2013) 015025 [arXiv:1304.1159] [INSPIRE].

[53] M. Chakraborti, U. Chattopadhyay, S. Rao and D.P. Roy, Higgsino Dark Matter in Nonuniversal Gaugino Mass Models, Phys. Rev. D 91 (2015) 035022 [arXiv:1411.4517] [INSPIRE]. 
[54] U. Chattopadhyay, D. Choudhury, M. Drees, P. Konar and D.P. Roy, Looking for a heavy Higgsino LSP in collider and dark matter experiments, Phys. Lett. B 632 (2006) 114 [hep-ph/0508098] [INSPIRE].

[55] U. Chattopadhyay and D.P. Roy, Higgsino dark matter in a SUGRA model with nonuniversal gaugino masses, Phys. Rev. D 68 (2003) 033010 [hep-ph/0304108] [INSPIRE].

[56] M. Chakraborti, U. Chattopadhyay and S. Poddar, How light a higgsino or a wino dark matter can become in a compressed scenario of MSSM, JHEP 09 (2017) 064 [arXiv: 1702.03954] [INSPIRE].

[57] MSSM Working Group collaboration, A. Djouadi et al., The Minimal supersymmetric standard model: Group summary report, hep-ph/9901246 [INSPIRE].

[58] M. Chakraborti, U. Chattopadhyay, A. Choudhury, A. Datta and S. Poddar, Reduced LHC constraints for higgsino-like heavier electroweakinos, JHEP 11 (2015) 050 [arXiv: 1507.01395] [INSPIRE].

[59] M. Chakraborti, U. Chattopadhyay, A. Choudhury, A. Datta and S. Poddar, The Electroweak Sector of the pMSSM in the Light of LHC - $8 \mathrm{TeV}$ and Other Data, JHEP 07 (2014) 019 [arXiv: 1404.4841] [InSPIRE].

[60] Muon G-2 collaboration, G.W. Bennett et al., Final Report of the Muon E821 Anomalous Magnetic Moment Measurement at BNL, Phys. Rev. D 73 (2006) 072003 [hep-ex/0602035] [INSPIRE].

[61] W. Gohn, The muon g-2 experiment at Fermilab, arXiv:1611.04964 [INSPIRE].

[62] A. Hoecker and W. Marciano, The Muon Anomalous Magnetic Moment, in Review of Particle Physics, Particle Data Group [Chin. Phys. C 40 (2016) 100001].

[63] M. Davier, A. Hoecker, B. Malaescu and Z. Zhang, Reevaluation of the hadronic vacuum polarisation contributions to the Standard Model predictions of the muon $g-2$ and $\alpha\left(m_{Z}^{2}\right)$ using newest hadronic cross-section data, Eur. Phys. J. C 77 (2017) 827 [arXiv:1706.09436] [INSPIRE].

[64] HFLAV collaboration, Y. Amhis et al., Averages of b-hadron, c-hadron and $\tau$-lepton properties as of summer 2016, Eur. Phys. J. C 77 (2017) 895 [arXiv:1612.07233] [INSPIRE].

[65] U. Haisch and F. Mahmoudi, MSSM: Cornered and Correlated, JHEP 01 (2013) 061 [arXiv: 1210.7806] [INSPIRE].

[66] U. Chattopadhyay and A. Dey, Exploring MSSM for Charge and Color Breaking and Other Constraints in the Context of Higgs@125 GeV, JHEP 11 (2014) 161 [arXiv:1409.0611] [INSPIRE].

[67] B. Bhattacherjee, M. Chakraborti, A. Chakraborty, U. Chattopadhyay, D. Das and D.K. Ghosh, Implications of the $98 \mathrm{GeV}$ and $125 \mathrm{GeV}$ Higgs scenarios in nondecoupling supersymmetry with updated ATLAS, CMS and PLANCK data, Phys. Rev. D 88 (2013) 035011 [arXiv: 1305.4020] [INSPIRE].

[68] B. Bhattacherjee, M. Chakraborti, A. Chakraborty, U. Chattopadhyay and D.K. Ghosh, Status of the 98-125 GeV Higgs bosons scenario with updated LHC-8 data, Phys. Rev. D 93 (2016) 075004 [arXiv: 1511.08461] [inSPIRE].

[69] C.S. Ün, Ş.H. Tanyıldızı, S. Kerman and L. Solmaz, Generalized Soft Breaking Leverage for the MSSM, Phys. Rev. D 91 (2015) 105033 [arXiv:1412.1440] [INSPIRE]. 
[70] U. Chattopadhyay and A. Dey, Probing Non-holomorphic MSSM via precision constraints, dark matter and LHC data, JHEP 10 (2016) 027 [arXiv:1604.06367] [INSPIRE].

[71] S.P. Martin, Dimensionless supersymmetry breaking couplings, flat directions and the origin of intermediate mass scales, Phys. Rev. D 61 (2000) 035004 [hep-ph/9907550] [INSPIRE].

[72] L.J. Hall and L. Randall, Weak scale effective supersymmetry, Phys. Rev. Lett. 65 (1990) 2939 [INSPIRE].

[73] I. Jack and D.R.T. Jones, Nonstandard soft supersymmetry breaking, Phys. Lett. B 457 (1999) 101 [hep-ph/9903365] [INSPIRE].

[74] I. Jack and D.R.T. Jones, Quasiinfrared fixed points and renormalization group invariant trajectories for nonholomorphic soft supersymmetry breaking, Phys. Rev. D 61 (2000) 095002 [hep-ph/9909570] [INSPIRE].

[75] J.M. Frere, M.V. Libanov and S.V. Troitsky, Neutrino masses from nonstandard supersymmetry breaking terms, Phys. Lett. B 479 (2000) 343 [hep-ph/9912204] [INSPIRE].

[76] J.P.J. Hetherington, The Spectrum of the MSSM with nonstandard supersymmetry breaking, JHEP 10 (2001) 024 [hep-ph/0108206] [INSPIRE].

[77] M.A. Cakir, S. Mutlu and L. Solmaz, Phenomenological issues in supersymmetry with non-holomorphic soft breaking, Phys. Rev. D 71 (2005) 115005 [hep-ph/0501286] [INSPIRE].

[78] A. Sabanci, A. Hayreter and L. Solmaz, Higgs Boson Masses Of The MSSM With General Soft Breaking, Phys. Lett. B 661 (2008) 154 [arXiv:0801.2029] [InSPIRE].

[79] E. Cincioglu, A. Hayreter, A. Sabanci and L. Solmaz, Loop corrections to the neutral Higgs bosons of the non-holomorphic supersymmetric standard model with explicit CP-violation, arXiv:0905.0726 [INSPIRE].

[80] G.G. Ross, K. Schmidt-Hoberg and F. Staub, On the MSSM Higgsino mass and fine tuning, Phys. Lett. B 759 (2016) 110 [arXiv:1603.09347] [INSPIRE].

[81] G.G. Ross, K. Schmidt-Hoberg and F. Staub, Revisiting fine-tuning in the MSSM, JHEP 03 (2017) 021 [arXiv:1701.03480] [INSPIRE].

[82] J. Beuria and A. Dey, Exploring Charge and Color Breaking vacuum in Non-Holomorphic MSSM, JHEP 10 (2017) 154 [arXiv:1708.08361] [INSPIRE].

[83] H.E. Haber and J.D. Mason, Hard supersymmetry-breaking 'wrong-Higgs' couplings of the MSSM, Phys. Rev. D 77 (2008) 115011 [arXiv:0711.2890] [INSPIRE].

[84] GAMBIT collaboration, P. Athron et al., Global fits of GUT-scale SUSY models with GAMBIT, Eur. Phys. J. C 77 (2017) 824 [arXiv:1705.07935] [INSPIRE].

[85] GAMBIT collaboration, P. Athron et al., A global fit of the MSSM with GAMBIT, Eur. Phys. J. C 77 (2017) 879 [arXiv:1705.07917] [InSPIRE].

[86] B. Bhattacherjee, A. Chakraborty and A. Choudhury, Status of the MSSM Higgs sector using global analysis and direct search bounds and future prospects at the High Luminosity LHC, Phys. Rev. D 92 (2015) 093007 [arXiv: 1504.04308] [INSPIRE].

[87] P. Bechtle et al., Constrained Supersymmetry after two years of LHC data: a global view with Fittino, JHEP 06 (2012) 098 [arXiv:1204.4199] [INSPIRE].

[88] M. Dine and W. Fischler, A Phenomenological Model of Particle Physics Based on Supersymmetry, Phys. Lett. B 110 (1982) 227 [INSPIRE]. 
[89] C.R. Nappi and B.A. Ovrut, Supersymmetric Extension of the $\mathrm{SU}(3) \times \mathrm{SU}(2) x U(1)$ Model, Phys. Lett. 113B (1982) 175 [INSPIRE].

[90] L. Álvarez-Gaumé, M. Claudson and M.B. Wise, Low-Energy Supersymmetry, Nucl. Phys. B 207 (1982) 96 [INSPIRE].

[91] M. Dine and A.E. Nelson, Dynamical supersymmetry breaking at low-energies, Phys. Rev. D 48 (1993) 1277 [hep-ph/9303230] [INSPIRE].

[92] M. Dine, A.E. Nelson and Y. Shirman, Low-energy dynamical supersymmetry breaking simplified, Phys. Rev. D 51 (1995) 1362 [hep-ph/9408384] [InSPIRE].

[93] M. Dine, A.E. Nelson, Y. Nir and Y. Shirman, New tools for low-energy dynamical supersymmetry breaking, Phys. Rev. D 53 (1996) 2658 [hep-ph/9507378] [INSPIRE].

[94] G.F. Giudice and R. Rattazzi, Theories with gauge mediated supersymmetry breaking, Phys. Rept. 322 (1999) 419 [hep-ph/9801271] [INSPIRE].

[95] G.F. Giudice and R. Rattazzi, Extracting supersymmetry breaking effects from wave function renormalization, Nucl. Phys. B 511 (1998) 25 [hep-ph/9706540] [INSPIRE].

[96] K. Jedamzik, M. Lemoine and G. Moultaka, Gravitino dark matter in gauge mediated supersymmetry breaking, Phys. Rev. D 73 (2006) 043514 [hep-ph/0506129] [InSPIRE].

[97] S. Dimopoulos, G.F. Giudice and A. Pomarol, Dark matter in theories of gauge mediated supersymmetry breaking, Phys. Lett. B 389 (1996) 37 [hep-ph/9607225] [INSPIRE].

[98] S. Dimopoulos, S.D. Thomas and J.D. Wells, Sparticle spectroscopy and electroweak symmetry breaking with gauge mediated supersymmetry breaking, Nucl. Phys. B 488 (1997) 39 [hep-ph/9609434] [INSPIRE].

[99] E.A. Baltz and H. Murayama, Gravitino warm dark matter with entropy production, JHEP 05 (2003) 067 [astro-ph/0108172] [INSPIRE].

[100] M. Fujii and T. Yanagida, Natural gravitino dark matter and thermal leptogenesis in gauge mediated supersymmetry breaking models, Phys. Lett. B 549 (2002) 273 [hep-ph/0208191] [INSPIRE].

[101] J.L. Feng, M. Kamionkowski and S.K. Lee, Light Gravitinos at Colliders and Implications for Cosmology, Phys. Rev. D 82 (2010) 015012 [arXiv: 1004.4213] [inSPIRE].

[102] W. Ahmed, L. Calibbi, T. Li, A. Mustafayev and S. Raza, Low Fine Tuning in Yukawa-deflected Gauge Mediation, Phys. Rev. D 95 (2017) 095031 [arXiv:1612.07125] [INSPIRE].

[103] D.M. Pierce, J.A. Bagger, K.T. Matchev and R.-j. Zhang, Precision corrections in the minimal supersymmetric standard model, Nucl. Phys. B 491 (1997) 3 [hep-ph/9606211] [INSPIRE].

[104] F. Staub, SARAH 3.2: Dirac Gauginos, UFO output and more, Comput. Phys. Commun. 184 (2013) 1792 [arXiv:1207.0906] [INSPIRE].

[105] F. Staub, SARAH 4 : A tool for (not only SUSY) model builders, Comput. Phys. Commun. 185 (2014) 1773 [arXiv: 1309.7223] [INSPIRE].

[106] F. Staub, Introduction to SARAH and related tools, PoS(CORFU2015) 027 [arXiv: 1509.07061] [INSPIRE].

[107] F. Staub, Tutorial to SARAH, PoS (CORFU2015) 058 [arXiv: 1603.05958] [INSPIRE]. 
[108] W. Porod, SPheno, a program for calculating supersymmetric spectra, SUSY particle decays and SUSY particle production at $e^{+} e^{-}$colliders, Comput. Phys. Commun. 153 (2003) 275 [hep-ph/0301101] [INSPIRE].

[109] F. Staub, From Superpotential to Model Files for FeynArts and CalcHep/CompHEP, Comput. Phys. Commun. 181 (2010) 1077 [arXiv:0909.2863] [InSPIRE].

[110] F. Staub, Automatic Calculation of supersymmetric Renormalization Group Equations and Self Energies, Comput. Phys. Commun. 182 (2011) 808 [arXiv:1002.0840] [InSPIRE].

[111] W. Porod and F. Staub, SPheno 3.1: Extensions including flavour, CP-phases and models beyond the MSSM, Comput. Phys. Commun. 183 (2012) 2458 [arXiv:1104.1573] [INSPIRE].

[112] W. Porod, F. Staub and A. Vicente, A Flavor Kit for BSM models, Eur. Phys. J. C 74 (2014) 2992 [arXiv: 1405.1434] [INSPIRE].

[113] S.P. Martin and M.T. Vaughn, Two loop renormalization group equations for soft supersymmetry breaking couplings, Phys. Rev. D 50 (1994) $2282[$ Erratum ibid. D 78 (2008) 039903] [hep-ph/9311340] [INSPIRE].

[114] M.D. Goodsell, K. Nickel and F. Staub, Two-Loop Higgs mass calculations in supersymmetric models beyond the MSSM with SARAH and SPheno, Eur. Phys. J. C 75 (2015) 32 [arXiv:1411.0675] [INSPIRE].

[115] M. Goodsell, K. Nickel and F. Staub, Generic two-loop Higgs mass calculation from a diagrammatic approach, Eur. Phys. J. C 75 (2015) 290 [arXiv:1503.03098] [InSPIRE].

[116] Particle Data Group collaboration, C. Patrignani et al., Review of Particle Physics, Chin. Phys. C 40 (2016) 100001.

[117] G. Degrassi, S. Heinemeyer, W. Hollik, P. Slavich and G. Weiglein, Towards high precision predictions for the MSSM Higgs sector, Eur. Phys. J. C 28 (2003) 133 [hep-ph/0212020] [INSPIRE].

[118] B.C. Allanach, A. Djouadi, J.L. Kneur, W. Porod and P. Slavich, Precise determination of the neutral Higgs boson masses in the MSSM, JHEP 09 (2004) 044 [hep-ph/0406166] [INSPIRE].

[119] S.P. Martin, Three-loop corrections to the lightest Higgs scalar boson mass in supersymmetry, Phys. Rev. D 75 (2007) 055005 [hep-ph/0701051] [INSPIRE].

[120] R.V. Harlander, P. Kant, L. Mihaila and M. Steinhauser, Higgs boson mass in supersymmetry to three loops, Phys. Rev. Lett. 100 (2008) 191602 [Phys. Rev. Lett. 101 (2008) 039901] [arXiv:0803.0672] [INSPIRE].

[121] S. Heinemeyer, O. Stal and G. Weiglein, Interpreting the LHC Higgs Search Results in the MSSM, Phys. Lett. B 710 (2012) 201 [arXiv:1112.3026] [INSPIRE].

[122] A. Arbey, M. Battaglia, A. Djouadi and F. Mahmoudi, The Higgs sector of the phenomenological MSSM in the light of the Higgs boson discovery, JHEP 09 (2012) 107 [arXiv:1207.1348] [INSPIRE].

[123] M. Chakraborti, U. Chattopadhyay and R.M. Godbole, Implication of a Higgs boson at $125 \mathrm{GeV}$ within the stochastic superspace framework, Phys. Rev. D 87 (2013) 035022 [arXiv: 1211.1549] [INSPIRE]. 
[124] A. Albaid and K.S. Babu, Higgs boson of mass $125 \mathrm{GeV}$ in GMSB models with messenger-matter mixing, Phys. Rev. D 88 (2013) 055007 [arXiv:1207.1014] [INSPIRE].

[125] Z. Kang, T. Li, T. Liu, C. Tong and J.M. Yang, A Heavy SM-like Higgs and a Light Stop from Yukawa-Deflected Gauge Mediation, Phys. Rev. D 86 (2012) 095020 [arXiv:1203.2336] [INSPIRE].

[126] P. Byakti and T.S. Ray, Burgeoning the Higgs mass to 125 GeV through messenger-matter interactions in GMSB models, JHEP 05 (2013) 055 [arXiv:1301.7605] [INSPIRE].

[127] J.A. Evans and D. Shih, Surveying Extended GMSB Models with $m_{h}=125 \mathrm{GeV}$, JHEP 08 (2013) 093 [arXiv: 1303.0228] [inSPIRE].

[128] J.L. Evans, M. Ibe and T.T. Yanagida, Relatively Heavy Higgs Boson in More Generic Gauge Mediation, Phys. Lett. B 705 (2011) 342 [arXiv:1107.3006] [INSPIRE].

[129] J.L. Lopez, D.V. Nanopoulos and X. Wang, Large (g-2)-mu in SU(5) x U(1) supergravity models, Phys. Rev. D 49 (1994) 366 [hep-ph/9308336] [INSPIRE].

[130] U. Chattopadhyay and P. Nath, Probing supergravity grand unification in the Brookhaven g-2 experiment, Phys. Rev. D 53 (1996) 1648 [hep-ph/9507386] [INSPIRE].

[131] T. Moroi, The Muon anomalous magnetic dipole moment in the minimal supersymmetric standard model, Phys. Rev. D 53 (1996) 6565 [Erratum ibid. D 56 (1997) 4424] [hep-ph/9512396] [INSPIRE].

[132] U. Chattopadhyay, D.K. Ghosh and S. Roy, Constraining anomaly mediated supersymmetry breaking framework via on going muon g-2 experiment at Brookhaven, Phys. Rev. D 62 (2000) 115001 [hep-ph/0006049] [INSPIRE].

[133] S. Heinemeyer, D. Stöckinger and G. Weiglein, Two loop SUSY corrections to the anomalous magnetic moment of the muon, Nucl. Phys. B 690 (2004) 62 [hep-ph/0312264] [InSPIRE].

[134] K. Kowalska, L. Roszkowski, E.M. Sessolo and A.J. Williams, GUT-inspired SUSY and the muon g-2 anomaly: prospects for LHC 14 TeV, JHEP 06 (2015) 020 [arXiv:1503.08219] [INSPIRE].

[135] G.-C. Cho, K. Hagiwara, Y. Matsumoto and D. Nomura, The MSSM confronts the precision electroweak data and the muon g-2, JHEP 11 (2011) 068 [arXiv:1104.1769] [INSPIRE].

[136] M. Endo, K. Hamaguchi, S. Iwamoto and K. Yanagi, Probing minimal SUSY scenarios in the light of muon $g-2$ and dark matter, JHEP 06 (2017) 031 [arXiv:1704.05287] [INSPIRE].

[137] J. Pradler, Electroweak Contributions to Thermal Gravitino Production, arXiv:0708.2786 [INSPIRE].

[138] L. Covi, J. Hasenkamp, S. Pokorski and J. Roberts, Gravitino Dark Matter and general neutralino NLSP, JHEP 11 (2009) 003 [arXiv:0908.3399] [INSPIRE].

[139] S. Ambrosanio, G.L. Kane, G.D. Kribs, S.P. Martin and S. Mrenna, Search for supersymmetry with a light gravitino at the Fermilab Tevatron and CERN LEP colliders, Phys. Rev. D 54 (1996) 5395 [hep-ph/9605398] [InSPIRE].

[140] S. Ambrosanio and G.A. Blair, Measuring gauge mediated supersymmetry breaking parameters at a 500-GeV $e^{+} e^{-}$linear collider, Eur. Phys. J. C 12 (2000) 287 [hep-ph/9905403] [INSPIRE]. 
[141] S. Bobrovskyi, J. Hajer and S. Rydbeck, Long-lived higgsinos as probes of gravitino dark matter at the LHC, JHEP 02 (2013) 133 [arXiv:1211.5584] [INSPIRE].

[142] M. Asano, T. Ito, S. Matsumoto and T. Moroi, Exploring Supersymmetric Model with Very Light Gravitino at the LHC, JHEP 03 (2012) 011 [arXiv:1111.3725] [INSPIRE].

[143] Y. Kats, P. Meade, M. Reece and D. Shih, The Status of GMSB After 1/fb at the LHC, JHEP 02 (2012) 115 [arXiv:1110.6444] [INSPIRE].

[144] S. Bobrovskyi, W. Buchmüller, J. Hajer and J. Schmidt, Quasi-stable neutralinos at the LHC, JHEP 09 (2011) 119 [arXiv:1107.0926] [INSPIRE].

[145] K. Ishiwata, T. Ito and T. Moroi, Long-Lived Unstable Superparticles at the LHC, Phys. Lett. B 669 (2008) 28 [arXiv:0807.0975] [INSPIRE]. 